\title{
Imperfect Preventive Maintenance Policies With Unpunctual Execution
}

\author{
Xiaolin Wang, Student Member, IEEE, Hang Zhou, Ajith Kumar Parlikad, Member, IEEE and Min \\ Xie, Fellow, IEEE
}

\begin{abstract}
Traditional maintenance planning problems usually presume that preventive maintenance (PM) policies will be executed exactly as planned. In reality, however, maintainers often deviate from the intended PM policy, resulting in unpunctual PM executions that may reduce maintenance effectiveness. This article studies two imperfect PM policies with unpunctual executions for infinite and finite planning horizons, respectively. Under the former policy, imperfect PM actions are periodically performed and the system is preventively replaced at the last PM instant. The objective is to determine the optimal number of PM actions and associated PM interval so as to minimize the long-run average cost rate. While the latter policy specifies that a system is subject to periodic PM activities within a finite planning horizon and there is no PM activity at the end of the horizon. The aim is then to identify the optimal number of PM activities to minimize the expected total maintenance cost. We discuss the modeling and optimization of the two unpunctual PM policies, and then explore the impact of unpunctual executions on the optimal PM decisions and corresponding maintenance expenses in an analytical or numerical way. The resulting insights are helpful for practitioners to adjust their PM plans when unpunctual executions are anticipated.
\end{abstract}

Index Terms-Imperfect preventive maintenance, unpunctual execution, hazard rate adjustment, infinite horizon, finite horizon.

\section{INTRODUCTION}

$\mathbf{P}$ REVENTIVE maintenance (PM) is a set of activities to be performed before system failures, aiming at keeping the system in a good working state and reducing its operational expenditure [1], [2]. However, it is not wise to maintain systems too frequently as this would incur excessive maintenance expenses. For this reason, determining an optimal PM policy (in terms of number of PM actions, PM intervals, etc.) to balance the benefits and costs of PM is an essential decisionmaking problem. The study of PM starts from the seminal work of Barlow and Hunter [3] and is still a vibrant research topic; see [4]-[9] for recent progresses.

Manuscript received September 23, 2019; revised February 12, 2020; accepted March 22, 2020. This work was supported by the National Natural Science Foundation of China (grant numbers 71532008, 71971181) and also by the Research Grants Council of Hong Kong under a Theme-based Research Fund (grant number T32-101/15-R) and a General Research Fund (grant number CityU 11203519). Associated Editor: Ming J. Zuo. (Corresponding author: Xiaolin Wang.)

X. Wang and M. Xie are with the Department of Systems Engineering and Engineering Management, City University of Hong Kong, Kowloon, Hong Kong SAR, and also with the City University of Hong Kong Shenzhen Research Institute, Shenzhen, China (e-mail: xlwang28-c@my.cityu.edu.hk; minxie@cityu.edu.hk).

H. Zhou and A. K. Parlikad are with the Institute for Manufacturing, Department of Engineering, University of Cambridge, Cambridge CB30FS, U.K. (e-mail: hz356@cam.ac.uk; aknp2@cam.ac.uk).
In practice, PM actions can be periodically or sequentially scheduled [10]. Under a periodic PM policy, a system is preventively maintained at integer multiples of a certain fixed interval; while a sequential policy specifies that a system is maintained at a sequence of unequal-length intervals. Generally, periodic PM is convenient to implement, whereas sequential PM is more effective for aging systems that require more frequent maintenance. On the other hand, the effect of a maintenance action on system reliability could be perfect, minimal or imperfect [11]. Perfect and minimal maintenance actions are two extremes: A perfect maintenance action is assumed to restore a failed system to an "as-good-as-new" state, whereas a minimal repair makes a failed system operational, with its state being that just before failure, i.e., the system is "as-bad-as-old" after a minimal repair [3]. A more realistic consideration is that the system state after maintenance is between "as-good-as-new" and "as-bad-as-old". This leads to the concept of imperfect maintenance [2], [11], [12]. Up to now, numerous models have been developed to formulate imperfect maintenance effects. In principle, existing models can be categorized by the measures that maintenance models manipulate: methods modifying the lifetime distribution (e.g., geometric process models [13], [14]), methods relating to the failure rate/intensity function (e.g., failure intensity reduction models [12], hazard rate adjustment models [15], [16]), methods reducing the system age [12], [17], and hybrid models [2], [18]. In this work, we focus on periodic, imperfect PM policies and adopt the hazard rate adjustment model.

In the literature, many studies deal with the optimization of imperfect PM policies for an infinite planning horizon. The objective is to determine optimal number of PM actions and PM interval (or a sequence of PM intervals) to minimize the longrun average cost rate. Lin et al. [18] developed sequential imperfect PM models with two failure modes-maintainable and non-maintainable. Wu and Clements-Croome [19] investigated two periodic imperfect PM policies with random PM quality. Zequeira and Bérenguer [20] studied an optimal periodic imperfect PM policy for a system with maintainable and nonmaintainable failure modes. Sheu and Chang [21] developed an extended periodic imperfect PM model with age-dependent failure types-Type I (minor) and Type II (catastrophic). Cha and Finkelstein [22] studied optimal imperfect periodic and age-based maintenance with long-run asymptotic virtual age. Sun et al. [23] developed a novel periodic PM model that involves the saturation effect of imperfect maintenance. Zhao et al. [24] investigated a condition-based inspectionreplacement policy for degrading systems. Moreover, there 
are also a few publications addressing both periodic and sequential imperfect PM policies. Nakagawa [10] studied both periodic and sequential PM policies based on the hazard rate adjustment PM model. Wang and Yam [13] and Wang et al. [14] investigated periodic and sequential PM policies based on generalized geometric processes.

In reality, the working spans of most systems are finite [16]. The maintenance planning horizon in this scenario is thus finite, and the decision-making problem is to identify optimal number of PM actions (and PM degree) to minimize the total expected maintenance cost over the finite horizon of interest. In the literature, there are quite a few studies on imperfect PM policies for a finite planning horizon, e.g., warranty period, lease period, and maintenance outsourcing period [25]. Nakagawa [16] provided an excellent summary of maintenance policies (including replacement, imperfect PM and inspection policies) for a finite horizon in a general context. Chien [26] developed an imperfect PM model for warranted systems with "worse-than-minimal" repair upon a failure. Shang et al. [27] studied optimal condition-based warranty design and post-warranty imperfect PM optimization for products subject to stochastic degradation. Su and Wang [28] and Wang et al. [29] investigated quasi-sequential imperfect PM strategies over warranty/lease periods: PM actions are periodically performed within a single phase and the PM intervals in different phases differ. Qiu et al. [30] developed a novel maintenance model for energy generation systems under a power purchase agreement so as to maximize the expected net revenue. Recently, Petchrompo et al. [31] proposed a valuebased approach to optimizing maintenance plans for a multiasset $k$-out-of- $N$ system over a long planning horizon.

The aforementioned studies, either in an infinite or finite planning-horizon setting, implicitly assume that scheduled PM activities are punctually implemented exactly as planned. In practice, however, there exists quite a few cases in which PM executions are not punctual. For instance, scheduled PM activities may be advanced by breaks in operations or by forecasted bad weather (e.g., for offshore wind turbines); conversely, maintenance actions may be postponed by busy operational schedules or by unavailability of maintenance resources. de Jonge et al. [32] showed that strategically postponing a PM action helps collect more information to reduce the lifetime distribution uncertainty, leading to potential cost benefits in the long run. Yang et al. [33] developed a two-phase PM model, where preventive replacement in the second phase is delayed to sufficiently utilize the system's remaining lifetime and facilitate replacement preparations. Scarf et al. [34] found that for a critical system, opportunistic inspections may offer an economic advantage against periodic inspections in certain cases. On the other hand, the working cycle of a system that executes successive jobs, might be variable. In this case, it is better to perform PM (that is originally scheduled during a working cycle) after the job is completed. This motivates socalled random replacement policies in, e.g., Zhao et al. [35] and Zhao et al. [36]. In a similar setting, Zhu et al. [37] studied a PM rescheduling problem in which the equipment may not be available for planned PM due to busy operational schedules.

In the PM studies above, the maintenance planner and executor are, in essence, the same and the planner can adjust (mostly postpone) maintenance activities according to operational schedules, weather conditions, or resource availability, etc. In practice, however, maintenance planning and execution might be separate in certain scenarios [38]. For example, in vehicle warranty and maintenance contexts, optimal PM schedule within the warranty period is recommended by the manufacturer, whereas the PM implementation relies on vehicle owners to return their vehicles to authorized maintenance centers [39]. The vehicle owners, however, may be unpunctual so that the PM activities are performed earlier or later than recommended. A similar scenario is that a maintenance planner specifies an optimal PM policy but relies on a group of crews to execute the maintenance operations [38]. In such scenarios, the potential unpunctuality-stemming from the separation of policy specification and implementation-is independent of the PM policy specified by the maintenance planner. As a consequence, it is better for maintenance planners to prescribe their PM policies in anticipation of unpunctual executions. He et al. [38] is, to the authors' knowledge, the first attempt in this context. Considering traditional age and periodic replacement policies, He et al. [38] determined optimal planned replacement instants by minimizing long-run average cost rates, in anticipation of replacement unpunctuality. Recently, Wang et al. [39] studied an unpunctual PM policy for repairable products sold with a two-dimensional warranty.

In this article, we aim to extend the investigation of unpunctual replacement policies in $\mathrm{He}$ et al. [38] to a more general imperfect PM scenario. The potential unpunctuality of maintainers is what causes actual PM instants to randomly deviate from the planned instants. The hazard rate adjustment model in [15], [16], [19] is adopted to formulate imperfect maintenance effects. We first discuss the modeling and optimization of imperfect PM policies in infinite and finite planning-horizon settings, and then explore the impact of unpunctual executions on the optimal PM decisions and corresponding maintenance expenses in an analytical or numerical way. Some managerial insights are obtained accordingly.

Overall, the main contributions of this work are four-fold:

(i) From the practical perspective, imperfect PM policies are studied in both infinite and finite planning-horizon settings, unlike Wang et al. [39] that only considers a finite warranty period.

(ii) From the mathematical modeling perspective, the unpunctual PM models generalize their punctual counterparts by assuming that the maintenance unpunctuality is governed by a specific probability distribution.

(iii) From the analysis perspective, the impacts of unpunctual executions on the optimal number and interval of imperfect PM actions are discussed in an analytical or numerical manner.

(iv) From the managerial perspective, this work provides some interesting insights that are helpful for practitioners to adjust their PM schedules when unpunctual executions are anticipated.

The remainder of the article is structured as follows. Sections II and III discuss the modeling and optimization of 
the unpunctual imperfect PM policies for infinite and finite planning horizons, respectively. Numerical studies are also conducted in these sections to demonstrate the models and results. Section IV concludes this article and suggests potential topics for future research. All technical proofs are presented in the Appendix.

\section{Model A: Infinite Planning-Horizon Setting}

The first model of interest is an unpunctual PM policy for an infinite planning horizon (hereafter termed "Model A"). We generalize the periodic imperfect PM model in Nakagawa [10] and $\mathrm{Wu}$ and Clements-Croome [19] by assuming that actual PM instants deviate from the scheduled instants in a probabilistic manner-namely, the PM deviations are governed by a specific probability distribution. In what follows, we first formulate the average long-run cost rates for the punctual and unpunctual execution scenarios, respectively, and then examine the optimization and policy comparison of the two scenarios. Numerical studies are also conducted to demonstrate the punctual and unpunctual PM policies.

\section{A. The Optimization Models}

The following assumptions are made to facilitate the modeling and analysis.

(i) When there is no maintenance action, the hazard rate of the system, $\lambda(t)$, is strictly increasing to $\infty$, with $\lambda(0)=0$.

(ii) The $j$ th $\mathrm{PM}$ interval is $T+Y_{j}$ for $j=1,2, \ldots, N$, and the system is preventively replaced at the $N$ th PM instant. Essentially, $T$ is the constant interval between two successive PM actions, and $Y_{j}, j=1,2, \ldots, N$, is the probabilistic deviation between the constant and actual intervals of the $j$ th $\mathrm{PM}\left(Y_{0}=0\right)$. We assume that $Y_{j}$ 's are independent and identically distributed (i.i.d.) random variables, with a cumulative distribution function $G(y), y \in[l, u]$, where $l$ and $u(l \leq u)$ are the smallest and largest values $Y_{j}$ can take.

(iii) The effect of imperfect PM actions on system reliability is described by the hazard rate adjustment model [15]. The hazard rate becomes $\lambda_{j}(t)=a_{j} \lambda_{j-1}(t), t>0$, after the $j$ th PM action if it was $\lambda_{j-1}(t)$ before the PM, where $a_{j}$ is the associated hazard rate adjustment factor. As a result, the system has a hazard rate $\lambda_{j}(t)=A_{j} \lambda(t)$ for $t \in\left(0, T+Y_{j+1}\right)$, where $1=a_{0}<a_{1} \leq a_{2} \leq$ $\cdots \leq a_{N-1}, A_{j}=\prod_{k=0}^{j} a_{k}$, and $1=A_{0}<A_{1}<$ $\cdots<A_{N-1}$. In essence, this model assumes that each PM action can reduce the hazard rate to zero and then the hazard rate function grows more quickly than it did before.

(iv) A replacement action brings the system to be as good as new. The system undergoes minimal repair upon a failure between two adjacent PM actions. The hazard rate remains unchanged after a minimal repair.

(v) The durations for imperfect PM, minimal repair, and replacement are very small in comparison with the mean time to failure, and thus negligible. (vi) The costs of an imperfect PM, a minimal repair, and a replacement are $c_{p}, c_{m}$, and $c_{r}$, respectively, with $c_{r}>$ $c_{m}$ and $c_{r}>c_{p}$.

Based on the assumptions above, if PM actions are always performed on time-that is, $Y_{j}=0$ for $j=1,2, \ldots, N$, then the long-run average cost rate is

$$
\widetilde{C}_{\mathcal{A}}(T, N)=\frac{c_{m} \sum_{j=1}^{N} A_{j-1} \Lambda(T)+(N-1) c_{p}+c_{r}}{N T},
$$

where $\Lambda(t)=\int_{0}^{t} \lambda(x) \mathrm{d} x$ is the cumulative hazard rate function. The optimization problem is to determine the optimal number of PM actions $\widetilde{N}^{*}$ and optimal PM interval $\widetilde{T}^{*}$ such that $\widetilde{C}_{\mathcal{A}}(T, N)$ is minimized.

Remark 1: If we take $N=1$, then model (1) reduces to the well-known periodic replacement with minimal repair model in Barlow and Proschan [40], and the long-run average cost rate becomes $\widetilde{C}_{\mathcal{A}}(T, N=1)=\left(c_{m} \Lambda(T)+c_{r}\right) / T$. On the other hand, if $a_{1}=\cdots=a_{N-1}=1$ and $c_{p}=c_{r}$, i.e., all PM actions are perfect replacements, then model (1) is also equivalent to the periodic replacement with minimal repair model. In this study, we consider $a_{j}>1, j=1,2, \ldots, N-1$, for imperfect PM consideration.

On the other hand, if PM actions are unpunctually implemented, then the long-run average cost rate can be formulated as

$$
\begin{aligned}
& C_{\mathcal{A}}(T, N) \\
& =\frac{c_{m} \sum_{j=1}^{N} \int_{l}^{u} A_{j-1} \Lambda\left(T+y_{j}\right) \mathrm{d} G\left(y_{j}\right)+(N-1) c_{p}+c_{r}}{\sum_{j=1}^{N} \int_{l}^{u}\left(T+y_{j}\right) \mathrm{d} G\left(y_{j}\right)} \\
& =\frac{c_{m} \sum_{j=1}^{N} \int_{l}^{u} A_{j-1} \Lambda\left(T+y_{j}\right) \mathrm{d} G\left(y_{j}\right)+(N-1) c_{p}+c_{r}}{N\left(T+\mu_{y}\right)},
\end{aligned}
$$

where $\mu_{y}=\int_{l}^{u} y_{j} \mathrm{~d} G\left(y_{j}\right)$ is the expected deviation of each PM. Likewise, the objective is to seek the optimal values of $N^{*}$ and $T^{*}$ to minimize $C_{\mathcal{A}}(T, N)$, when the maintenance unpunctuality is anticipated. It should be noted that as $Y_{j}$ can take negative values, this model requires the feasible domain of $T$ to be $\{T \mid T>\max \{-l, 0\}\}$, so as to guarantee $T+Y_{j}>0$ for $j=1,2, \ldots, N$.

Remark 2: If we take $N=1$, then model (2) is simplified to the so-called "unpunctual age replacement with minimal repair" model in $\mathrm{He}$ et al. [38], and the long-run average cost rate becomes

$$
C_{\mathcal{A}}(T, N=1)=\frac{c_{m} \int_{l}^{u} \Lambda\left(T+y_{j}\right) \mathrm{d} G\left(y_{j}\right)+c_{r}}{T+\mu_{y}} .
$$

\section{B. Optimal PM Decisions}

In this subsection, we examine the optimal infinite-horizon PM decisions in the punctual and unpunctual scenarios, respectively, which form the foundation of further analyses in Section II-C.

We first investigate the optimal $N^{*}$ and $T^{*}$ of the imperfect PM policy with unpunctual executions. For any feasible PM interval $T>\max \{-l, 0\}$, the optimal $N^{*}$ can be obtained by 
solving two inequalities, namely, $C_{\mathcal{A}}(T, N-1)>C_{\mathcal{A}}(T, N)$ and $C_{\mathcal{A}}(T, N+1) \geq C_{\mathcal{A}}(T, N)$, which yields

$$
\mathcal{J}_{\mathcal{A}}(T, N-1)<\frac{c_{r}-c_{p}}{c_{m}} \text { and } \mathcal{J}_{\mathcal{A}}(T, N) \geq \frac{c_{r}-c_{p}}{c_{m}},
$$

where

$$
\begin{aligned}
\mathcal{J}_{\mathcal{A}}(T, N)= & N \int_{l}^{u} A_{N} \Lambda\left(T+y_{N+1}\right) \mathrm{d} G\left(y_{N+1}\right) \\
& -\sum_{j=1}^{N} \int_{l}^{u} A_{j-1} \Lambda\left(T+y_{j}\right) \mathrm{d} G\left(y_{j}\right),
\end{aligned}
$$

for $N=1,2, \ldots$, and $\mathcal{J}_{\mathcal{A}}(T, 0)=0$.

Proposition 1: For any feasible $T>\max \{-l, 0\}$, there exists a finite and unique solution $N^{*}$ to (3), which minimizes the average cost rate $C_{\mathcal{A}}(T, N)$.

Now, suppose that the number of PM actions $N \geq 1$ is fixed, we attempt to identify the optimal $T^{*}$ that minimizes $C_{\mathcal{A}}(T, N)$. To this end, we differentiate $C_{\mathcal{A}}(T, N)$ with respect to $T$ and set $\partial C_{\mathcal{A}}(T, N) / \partial T=0$. Then, we have

$$
\sum_{j=1}^{N} \mathcal{L}_{\mathcal{A}, i}(T)=\frac{(N-1) c_{p}+c_{r}}{c_{m}}
$$

where

$$
\begin{aligned}
\mathcal{L}_{\mathcal{A}, i}(T)= & \left(T+\mu_{y}\right) \int_{l}^{u} A_{j-1} \lambda\left(T+y_{j}\right) \mathrm{d} G\left(y_{j}\right) \\
& -\int_{l}^{u} A_{j-1} \Lambda\left(T+y_{j}\right) \mathrm{d} G\left(y_{j}\right) .
\end{aligned}
$$

The following proposition can be obtained regarding the optimal $T^{*}$ for any fixed $N \geq 1$.

Proposition 2: For any finite $N \geq 1$, if

$$
\lim _{T \rightarrow \max \{-l, 0\}} \sum_{j=1}^{N} \mathcal{L}_{\mathcal{A}, i}(T)<\frac{(N-1) c_{p}+c_{r}}{c_{m}},
$$

then there exists a unique solution $T^{*}$ to (4), and the resultant cost rate is

$$
C_{\mathcal{A}}\left(T^{*}, N\right)=\frac{c_{m}}{N} \sum_{j=1}^{N} \int_{l}^{u} A_{j-1} \lambda\left(T^{*}+y_{j}\right) \mathrm{d} G\left(y_{j}\right) ;
$$

otherwise,

$$
\inf C_{\mathcal{A}}(T, N)=\lim _{T \rightarrow \max \{-l, 0\}} C_{\mathcal{A}}(T, N) .
$$

Note that condition (5) guarantees the uniqueness of the solution $T^{*}$ to (4). To satisfy this condition, $\left[(N-1) c_{p}+\right.$ $\left.c_{r}\right] / c_{m}$ should be relatively large, which indicates that the imperfect PM cost and the replacement cost should be large in comparison with the minimal repair cost. In this work, we suppose that condition (5) will hold, at least for the optimal $N^{*}$, throughout the remainder of Section II.

According to the analysis above, we have to solve (3) and (4) simultaneously, when determining the optimal $N^{*}$ and $T^{*}$ for Model A with unpunctual executions. To facilitate this process, we develop an enumeration algorithm to search $N^{*}$ and $T^{*}$ that minimize $C_{\mathcal{A}}(T, N)$; see Algorithm 1. For a fixed $N$ (starting from $N=1$ ), if condition (5) is satisfied, then a unique optimal PM interval $T$ can be obtained by solving (4).

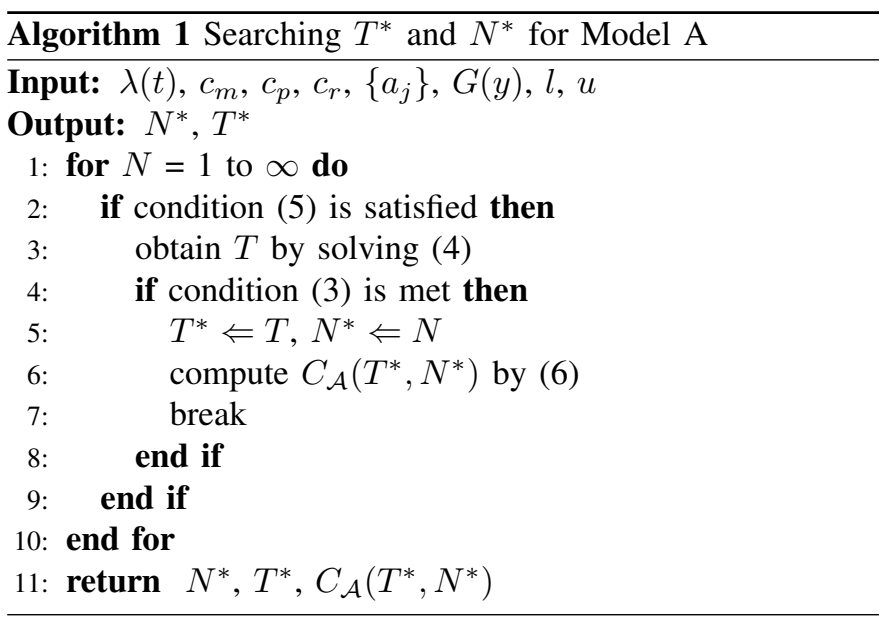

We then turn to check condition (3) for current $N$ and $T$. If the inequalities well hold, then the current $N$ and $T$ are optimal; otherwise, set $N \leftarrow N+1$, and repeat the procedure. The analytical results in Propositions 1 and 2 guarantee that the algorithm will stop at a finite $N^{*}$, with the associated optimal $T^{*}$ obtained.

Next, we look at the optimal decisions $\widetilde{N}^{*}$ and $\widetilde{T}^{*}$ of the periodic PM policy with punctual executions. As the optimization procedure is quite similar to the unpunctual scenario, we directly present the following result without detailed proof.

Proposition 3: For the infinite-horizon imperfect PM policy (Model A) with punctual executions, the optimal $\widetilde{N}^{*}$ and $\widetilde{T}^{*}$ exist when conditions (8) and (9) below are satisfied, simultaneously:

$$
\widetilde{\mathcal{J}}_{\mathcal{A}}(T, N-1)<\frac{c_{r}-c_{p}}{c_{m}} \text { and } \widetilde{\mathcal{J}}_{\mathcal{A}}(T, N) \geq \frac{c_{r}-c_{p}}{c_{m}},
$$

and

$$
\sum_{j=1}^{N} \widetilde{\mathcal{L}}_{\mathcal{A}, i}(T)=\frac{(N-1) c_{p}+c_{r}}{c_{m}},
$$

and the resulting average cost rate is

$$
\widetilde{C}_{\mathcal{A}}\left(\widetilde{T}^{*}, \widetilde{N}^{*}\right)=\frac{c_{m}}{\widetilde{N}^{*}} \sum_{j=1}^{\widetilde{N}^{*}} A_{j-1} \lambda\left(\widetilde{T}^{*}\right),
$$

where $\widetilde{\mathcal{L}}_{\mathcal{A}, i}(T)=A_{j-1}(T \lambda(T)-\Lambda(T)), \widetilde{\mathcal{J}}_{\mathcal{A}}(T, N)=$ $\left(N A_{N}-\sum_{j=1}^{N} A_{j-1}\right) \Lambda(T), N=1,2, \ldots$, and $\widetilde{\mathcal{J}}_{\mathcal{A}}(T, 0)=0$.

The optimal decision variables $\widetilde{N}^{*}$ and $\widetilde{T}^{*}$ in the punctual execution scenario can also be searched via Algorithm 1, with a slight modification.

\section{Impact of Unpunctual Executions on The Optimal PM Decisions}

In this subsection, we investigate the impact of unpunctual policy executions on the optimal PM decisions. For this purpose, we compare $\left(\widetilde{T}^{*}, \widetilde{N}^{*}\right)$ with $\left(T^{*}, N^{*}\right)$ under different conditions on the PM deviations. More precisely, two different scenarios of maintenance unpunctuality - that is, PM actions are never performed earlier (or later) than intended-are examined; see Theorems 1 and 2, respectively. Note that only convex hazard rate $\lambda(t)$ is considered below, as it is 
commonly used to characterize time-to-failure distributions for aging systems. Since it is difficult to derive analytical results for the case in which $\lambda(t)$ is concave increasing, we resort to numerical experiments instead.

Theorem 1: If $\lambda(t)$ is convex and $0 \leq l<u$, then we have the following results:

(i) if the two policies adopt the same $N \geq 1$, and

$$
\lim _{T \rightarrow 0} \mathcal{L}_{\mathcal{A}, i}(T) \geq 0
$$

then $T^{*}<\widetilde{T}^{*}$

(ii) if the two policies adopt the same $T>0$, then $N^{*} \leq$ $\widetilde{N}^{*}$.

Theorem 1 implies that if a fixed number $N(N \geq 1)$ of PM actions are scheduled and all of them are never performed earlier than intended, then the maintenance planner should schedule PM earlier than that in the punctual scenario; on the other hand, if a fixed PM interval $T(T>0)$ is set and all PM actions are never performed earlier than intended, then a fewer number of PM actions should be planned than that in the punctual scenario. Note that the inequality $N^{*} \leq \widetilde{N}^{*}$ is not strict because $N$ is an integer in nature, thus there is a possibility that $N^{*}=\widetilde{N}^{*}$, especially when the values of $\mathcal{J}_{\mathcal{A}}(T, N)$ and $\widetilde{\mathcal{J}}_{\mathcal{A}}(T, N)$ are close.

Theorem 2: If $\lambda(t)$ is convex and $l<u \leq 0$, then we have the following results:

(i) if the two policies adopt the same $N \geq 1$, then $T^{*}>\widetilde{T}^{*}$;

(ii) if the two policies adopt the same $T>-l$, then $N^{*} \geq$ $\widetilde{N}^{*}$.

In contrast, Theorem 2 indicates that if a fixed number $N$ $(N \geq 1)$ of $\mathrm{PM}$ actions are planned and they are always performed earlier than intended, then the maintenance planner should schedule PM later than that in the punctual scenario; while if a fixed PM interval $T(T>0)$ is specified and all PM actions are always performed earlier than intended, then a larger number of PM actions should be planned than that in the punctual scenario. Comparing Theorem 2 with Theorem 1 , we find that the scenario of unpunctual execution indeed has an impact on the optimal PM decisions (in terms of PM interval and number of PM actions).

Remark 3: For scenario (i) in Theorem 2 (i.e., the two policies adopt the same $N \geq 1$ ), there are two possible cases about the relationship of $\widetilde{T}^{*}$ and $-l$, i.e.,

(a) if $\sum_{j=1}^{N} \widetilde{\mathcal{L}}_{\mathcal{A}, i}(-l) \geq \frac{(N-1) c_{p}+c_{r}}{c_{m}}$, then $T^{*}>-l>\widetilde{T}^{*}$;

(b) if $\sum_{j=1}^{N} \widetilde{\mathcal{L}}_{\mathcal{A}, i}(-l)<\frac{(N-1) c_{p}+c_{r}}{c_{m}}$, then $T^{*}>\widetilde{T}^{*}>-l$.

The arguments in Remark 3 can be referred to the proof of Theorem 2 in the Appendix. This result originates from the different feasible domains of PM interval $T$ in punctual and unpunctual scenarios. Specifically, when $l<u \leq 0$, the feasible domain of PM interval $T$ in the unpunctual scenario is $\{T \mid T>-l\}$, whereas that in the punctual scenario is still $\{T \mid T>0\}$.

It is worth emphasizing that the results in Theorems 1 and 2 are either for fixed $N$ or for fixed $T$. The ideal comparison we attempt to perform is comparing $\left(\widetilde{T}^{*}, \widetilde{N}^{*}\right)$ with $\left(T^{*}, N^{*}\right)$ in their optimal values. However, such comparison is difficult, if not impossible, to conduct and thus we resort to numerical experiments again.
TABLE I

Optimal PM Decisions Under Punctual Executions in The INFINITE HORIZON SETTING.

\begin{tabular}{lccccccc}
\hline & \multicolumn{3}{c}{$\beta=1.5$} & & \multicolumn{3}{l}{$\beta=3.5$} \\
\cline { 2 - 3 } \cline { 6 - 7 }$c_{p}$ & $\tilde{N}^{*}$ & $\widetilde{T}^{*}$ & $\widetilde{C}_{\mathcal{A}}$ & & $\widetilde{N}^{*}$ & $\widetilde{T}^{*}$ & $\widetilde{C}_{\mathcal{A}}$ \\
\hline 0.1 & 4 & 74.53 & 0.305 & & 15 & 8.38 & 0.350 \\
0.5 & 4 & 76.48 & 0.309 & & 13 & 9.93 & 0.390 \\
1 & 4 & 78.89 & 0.314 & & 12 & 11.02 & 0.434 \\
2 & 4 & 83.60 & 0.323 & & 10 & 13.21 & 0.509 \\
5 & 3 & 117.12 & 0.341 & & 8 & 16.63 & 0.684 \\
\hline
\end{tabular}

\section{Numerical Studies}

In this subsection, numerical studies are presented to demonstrate the unpunctual PM policy for an infinite planning horizon, and to examine the impact of unpunctual executions on the optimal PM decisions.

Suppose that the system lifetime is Weibull distributed, i.e., $\lambda(t)=(\beta / \alpha)(t / \alpha)^{\beta-1}$ and $\Lambda(t)=(t / \alpha)^{\beta}$, with $\alpha=15$. For the shape parameter, only $\beta>1$ is considered, as it is well known that carrying out PM activities is uneconomic for $\beta \leq 1$ (the hazard rate function is decreasing or constant). In this study, we examine two typical shapes of hazard rate-concave $(1<\beta<2)$ and convex $(\beta>2)$. Specifically, $\beta=1.5$ and $\beta=3.5$ are considered for concave and convex hazard rates, respectively. The hazard rate adjustment factor is assumed to be $a_{j}=(5 j+1) /(4 j+1)$, i.e., $a_{j}$ is strictly increasing in $j$, from $a_{0}=1$ to $a_{\infty}=1.25$. Further assume that the minimal repair cost is $c_{m}=1$, and the replacement cost is $c_{r}=30$.

We first look at the imperfect PM policy in the punctual execution scenario. Table I summarizes the optimal PM decisions and corresponding average cost rates for different values of $c_{p}$. It can be seen that as the PM cost $c_{p}$ increases, the optimal number of PM actions $\widetilde{N}^{*}$ decreases, whereas both the optimal PM interval $\widetilde{T}^{*}$ and the cost rate $\widetilde{C}_{\mathcal{A}}\left(\widetilde{N}^{*}, \widetilde{T}^{*}\right)$ increase. This observation applies to both concave and convex hazard rates.

We then study the imperfect PM policy in the unpunctual execution scenario. For illustrative purposes, we assume that the PM deviation $Y_{j}$ has a uniform distribution $U(l, u)$, though our model accommodates to any feasible distribution of $Y_{j}$. To examine the impact of maintenance unpunctuality, we consider three scenarios of unpunctual executions-advanced $(l<u \leq$ $0)$, delayed $(0 \leq l<u)$, and hybrid $(l<0<u)$, coupled with three unpunctuality magnitudes (measured by $u-l$ ). Specifically, the three unpunctuality magnitudes correspond to $u-l=1, u-l=2$, and $u-l=5$, respectively. Tables II and III report the optimal PM decisions and corresponding average cost rates under unpunctual executions for $\beta=1.5$ and $\beta=$ 3.5 , respectively. In this tables, we define $\Psi=\left(T^{*}-\widetilde{T}^{*}\right) / \widetilde{T}^{*}$ and $\Omega=\left(C_{\mathcal{A}}\left(N^{*}, T^{*}\right)-\widetilde{C}_{\mathcal{A}}\left(\widetilde{N}^{*}, \widetilde{T}^{*}\right)\right) / \widetilde{C}_{\mathcal{A}}\left(\widetilde{N}^{*}, \widetilde{T}^{*}\right)$ as the relative changes of PM interval and cost rate, respectively, when comparing unpunctual PM policy with its punctual counterpart. It is clear that a positive (resp. negative) $\Psi$ corresponds to the case that $T^{*}>\widetilde{T}^{*}$ (resp. $T^{*}<\widetilde{T}^{*}$ ); and a larger (resp. smaller) absolute value of $\Psi$ implies a larger (resp. smaller) difference between $T^{*}$ and $\widetilde{T}^{*}$. The same argument applies to $\Omega$ as well. Figs. 1 and 2 further illustrate the values 
TABLE II

Optimal PM Decisions Under UnPUnCtual EXECUTIONS IN THE INFINITE Horizon SETTINg $(\beta=1.5)$.

\begin{tabular}{|c|c|c|c|c|c|c|c|c|c|c|c|c|c|c|c|}
\hline \multirow[b]{2}{*}{$c_{p}$} & \multicolumn{5}{|c|}{$l=0, u=1$} & \multicolumn{5}{|c|}{$l=0, u=2$} & \multicolumn{5}{|c|}{$l=0, u=5$} \\
\hline & $N^{*}$ & $T^{*}$ & $\Psi(\%)$ & $C_{\mathcal{A}}$ & $\Omega(\%)$ & $N^{*}$ & $T^{*}$ & $\Psi(\%)$ & $C_{\mathcal{A}}$ & $\Omega(\%)$ & $N^{*}$ & $T^{*}$ & $\Psi(\%)$ & $C_{\mathcal{A}}$ & $\Omega(\%)$ \\
\hline 0.1 & 4 & 74.03 & -0.67 & 0.305 & 0.00 & 4 & 73.53 & -1.34 & 0.305 & 0.00 & 4 & 72.05 & -3.33 & 0.305 & 0.00 \\
\hline 0.5 & 4 & 75.98 & -0.65 & 0.309 & 0.00 & 4 & 75.49 & -1.29 & 0.309 & 0.00 & 4 & 74.00 & -3.24 & 0.309 & 0.00 \\
\hline 1 & 4 & 78.39 & -0.63 & 0.314 & 0.00 & 4 & 77.90 & -1.25 & 0.314 & 0.00 & 4 & 76.41 & -3.14 & 0.314 & 0.00 \\
\hline 2 & 4 & 83.10 & -0.60 & 0.323 & 0.00 & 4 & 82.61 & -1.18 & 0.323 & 0.00 & 4 & 81.12 & -2.97 & 0.323 & 0.00 \\
\hline \multirow[t]{2}{*}{5} & 3 & 116.62 & -0.43 & 0.342 & 0.00 & 3 & 116.12 & -0.85 & 0.342 & 0.00 & 3 & 114.63 & -2.13 & 0.342 & 0.00 \\
\hline & \multicolumn{5}{|c|}{$l=-0.5, u=0.5$} & \multicolumn{5}{|c|}{$l=-1, u=1$} & \multicolumn{5}{|c|}{$l=-2.5, u=2.5$} \\
\hline$c_{p}$ & $N^{*}$ & $T^{*}$ & $\Psi(\%)$ & $C_{\mathcal{A}}$ & $\Omega(\%)$ & $N^{*}$ & $T^{*}$ & $\Psi(\%)$ & $C_{\mathcal{A}}$ & $\Omega(\%)$ & $N^{*}$ & $T^{*}$ & $\Psi(\%)$ & $C_{\mathcal{A}}$ & $\Omega(\%)$ \\
\hline 0.1 & 4 & 74.53 & 0.00 & 0.305 & 0.00 & 4 & 74.53 & 0.00 & 0.305 & 0.00 & 4 & 74.55 & 0.03 & 0.305 & 0.00 \\
\hline 0.5 & 4 & 76.48 & 0.00 & 0.309 & 0.00 & 4 & 76.49 & 0.01 & 0.309 & 0.00 & 4 & 76.50 & 0.03 & 0.309 & 0.00 \\
\hline 1 & 4 & 78.89 & 0.00 & 0.314 & 0.00 & 4 & 78.90 & 0.01 & 0.314 & 0.00 & 4 & 78.91 & 0.03 & 0.314 & 0.00 \\
\hline 2 & 4 & 83.60 & 0.00 & 0.323 & 0.00 & 4 & 83.61 & 0.01 & 0.323 & 0.00 & 4 & 83.62 & 0.02 & 0.323 & 0.00 \\
\hline \multirow[t]{2}{*}{5} & 3 & 117.12 & 0.00 & 0.342 & 0.00 & 3 & 117.12 & 0.00 & 0.342 & 0.00 & 3 & 117.13 & 0.01 & 0.342 & 0.00 \\
\hline & \multicolumn{5}{|c|}{$l=-1, u=0$} & \multicolumn{5}{|c|}{$l=-2, u=0$} & \multicolumn{5}{|c|}{$l=-5, u=0$} \\
\hline$c_{p}$ & $N^{*}$ & $T^{*}$ & $\Psi(\%)$ & $C_{\mathcal{A}}$ & $\Omega(\%)$ & $N^{*}$ & $T^{*}$ & $\Psi(\%)$ & $C_{\mathcal{A}}$ & $\Omega(\%)$ & $N^{*}$ & $T^{*}$ & $\Psi(\%)$ & $C_{\mathcal{A}}$ & $\Omega(\%)$ \\
\hline 0.1 & 4 & 75.03 & 0.67 & 0.305 & 0.00 & 4 & 75.53 & 1.34 & 0.305 & 0.00 & 4 & 77.05 & 3.38 & 0.305 & 0.00 \\
\hline 0.5 & 4 & 76.98 & 0.65 & 0.309 & 0.00 & 4 & 77.49 & 1.32 & 0.309 & 0.00 & 4 & 79.00 & 3.29 & 0.309 & 0.00 \\
\hline 1 & 4 & 79.39 & 0.63 & 0.314 & 0.00 & 4 & 79.90 & 1.28 & 0.314 & 0.00 & 4 & 81.41 & 3.19 & 0.314 & 0.00 \\
\hline 2 & 4 & 84.10 & 0.60 & 0.323 & 0.00 & 4 & 84.61 & 1.21 & 0.323 & 0.00 & 4 & 86.12 & 3.01 & 0.323 & 0.00 \\
\hline 5 & 3 & 117.62 & 0.43 & 0.342 & 0.00 & 3 & 118.12 & 0.85 & 0.342 & 0.00 & 3 & 119.63 & 2.14 & 0.342 & 0.00 \\
\hline
\end{tabular}
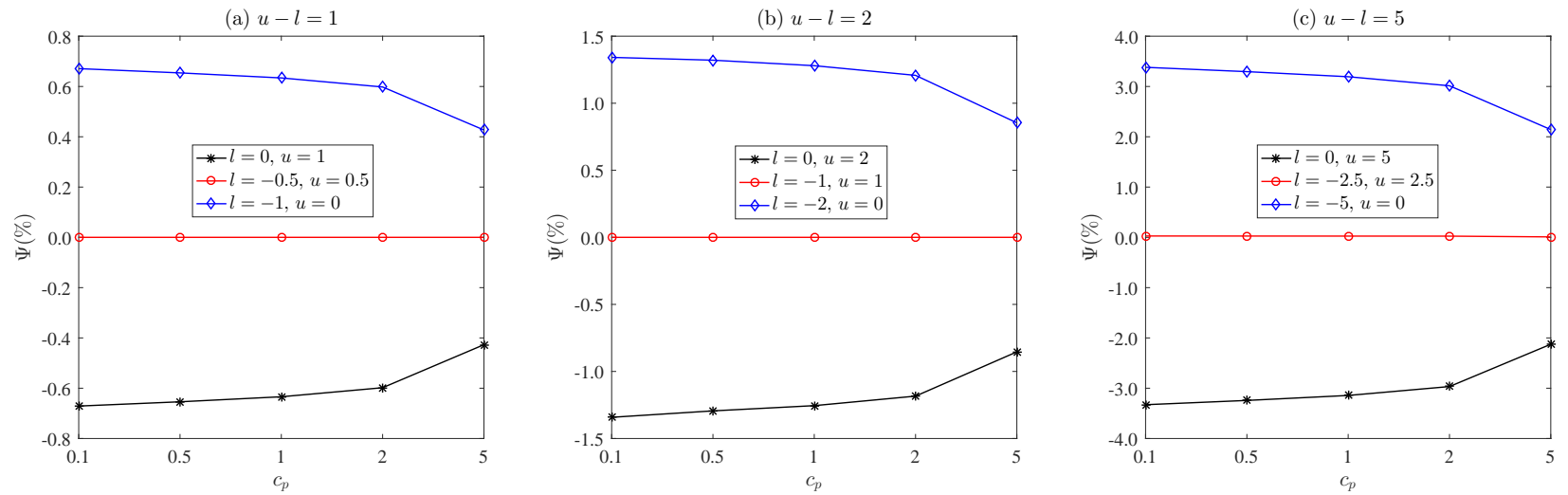

Fig. 1. Relative PM-Interval Change $\Psi$ in The Infinite Horizon Setting $(\beta=1.5)$.

of $\Psi$ for $\beta=1.5$ and $\beta=3.5$, respectively.

From these tables and figures, the following observations can be drawn:

(1) In the unpunctual PM policy, as the PM cost $c_{p}$ increases, the optimal number of PM actions $N^{*}$ decreases, whereas both the optimal PM interval $T^{*}$ and the average cost rate $C_{\mathcal{A}}\left(N^{*}, T^{*}\right)$ increase. This finding, similar to that for the punctual policy, applies to all numerical cases in Tables II and III, regardless of the unpunctuality scenarios and magnitudes.

(2) The unpunctual PM policy requires the same number of PM actions as the punctual policy for $\beta=1.5$ (see Table II); however, it needs slightly fewer number of PM actions for $\beta=3.5$, especially when the magnitude of maintenance unpunctuality, $u-l$, is large (see Table III). Moreover, Table III shows that the difference between $N^{*}$ and $\widetilde{N}^{*}$, in the case of $\beta=3.5$, depends more on the unpunctuality magnitude, rather than the unpunctuality scenario (advanced, delayed, or hybrid). This appears to be inconsistent with Theorems 1 and 2. Indeed, it is not the case, because the optimal PM intervals $T^{*}$ and $\widetilde{T}^{*}$ are not identical.

(3) It is interesting to observe that the optimal PM interval of the unpunctual PM policy can be shorter or longer than that of the punctual policy, depending on the unpunctuality scenario and magnitude (see Figs. 1 and 2). More specifically, (i) when PM actions are never performed earlier than intended $(0 \leq l<u), T^{*}<\widetilde{T}^{*}$; (ii) when PM actions are never performed later than intended $(l<u \leq 0), T^{*}>\widetilde{T}^{*}$; while (iii) when the unpunctuality scenario is hybrid $(l<0<u)$, the values of $T^{*}$ and $\widetilde{T}^{*}$ are close, especially when $u-l$ is not large. Observations (i) and (ii) well support the results in Theorems 1 and 2, although we cannot analytically prove the result for concave hazard rate. Furthermore, the difference between $T^{*}$ and $\widetilde{T}^{*}$ (measured by the 
TABLE III

Optimal PM Decisions Under Unpunctual Executions in The Infinite Horizon SETting $(\beta=3.5)$.

\begin{tabular}{|c|c|c|c|c|c|c|c|c|c|c|c|c|c|c|c|}
\hline \multirow[b]{2}{*}{$c_{p}$} & \multicolumn{5}{|c|}{$l=0, u=1$} & \multicolumn{5}{|c|}{$l=0, u=2$} & \multicolumn{5}{|c|}{$l=0, u=5$} \\
\hline & $N^{*}$ & $T^{*}$ & $\Psi(\%)$ & $C_{\mathcal{A}}$ & $\Omega(\%)$ & $N^{*}$ & $T^{*}$ & $\Psi(\%)$ & $C_{\mathcal{A}}$ & $\Omega(\%)$ & $N^{*}$ & $T^{*}$ & $\Psi(\%)$ & $C_{\mathcal{A}}$ & $\Omega(\%)$ \\
\hline 0.1 & 15 & 7.88 & -5.97 & 0.350 & 0.14 & 15 & 7.37 & -12.05 & 0.352 & 0.60 & 14 & 6.38 & -23.87 & 0.362 & 3.43 \\
\hline 0.5 & 13 & 9.43 & -5.04 & 0.391 & 0.10 & 15 & 8.92 & -10.17 & 0.392 & 0.4 & 13 & 7.38 & -25.68 & 0.401 & 2.66 \\
\hline 1 & 12 & 10.51 & -4.63 & 0.435 & 0.09 & 12 & 10.01 & -9.17 & 0.435 & 0.35 & 11 & 9.16 & -16.88 & 0.443 & 2.10 \\
\hline 2 & 10 & 12.71 & -3.79 & 0.509 & 0.06 & 10 & 12.20 & -7.65 & 0.510 & 0.24 & 10 & 10.67 & -19.23 & 0.516 & 1.49 \\
\hline \multirow[t]{2}{*}{5} & 8 & 16.12 & -3.07 & 0.684 & 0.03 & 8 & 16.53 & -0.60 & 0.685 & 0.13 & 7 & 15.01 & -9.74 & 0.690 & 0.85 \\
\hline & \multicolumn{5}{|c|}{$l=-0.5, u=0.5$} & \multicolumn{5}{|c|}{$l=-1, u=1$} & \multicolumn{5}{|c|}{$l=-2.5, u=2.5$} \\
\hline$c_{p}$ & $N^{*}$ & $T^{*}$ & $\Psi(\%)$ & $C_{\mathcal{A}}$ & $\Omega(\%)$ & $N^{*}$ & $T^{*}$ & $\Psi(\%)$ & $C_{\mathcal{A}}$ & $\Omega(\%)$ & $N^{*}$ & $T^{*}$ & $\Psi(\%)$ & $C_{\mathcal{A}}$ & $\Omega(\%)$ \\
\hline 0.1 & 15 & 8.38 & 0 & 0.350 & 0.14 & 15 & 8.3 & -0.12 & 0.352 & 0.6 & 1 & 8.8 & 5. & 0.362 & 3.43 \\
\hline 0.5 & 13 & 9.93 & 0.00 & 0.391 & 0.10 & 13 & 9.9 & -0.10 & 0.392 & 0.44 & 13 & 9.8 & -0.50 & 0.401 & 2.66 \\
\hline 1 & 12 & 11.01 & -0.09 & 0.435 & 0.09 & 12 & 11. & -0.09 & 0.436 & 0.35 & 11 & 11 & & 0.443 & 2.10 \\
\hline 2 & 10 & 13.21 & 0.00 & 0.509 & 0.06 & 10 & 13. & -0.08 & 0.510 & 0.24 & 10 & 13 & -0.30 & 0.516 & 1.49 \\
\hline \multirow[t]{2}{*}{5} & 8 & 16.62 & -0.06 & 0.684 & 0.03 & 8 & 17.53 & 5.41 & 0.685 & 0.13 & 7 & 17.51 & 5.29 & 0.690 & 0.85 \\
\hline & \multicolumn{5}{|c|}{$l=-1, u=0$} & \multicolumn{5}{|c|}{$l=-2, u=0$} & \multicolumn{5}{|c|}{$l=-5, u=0$} \\
\hline$c_{p}$ & $N^{*}$ & $T^{*}$ & $\Psi(\%)$ & $C_{\mathcal{A}}$ & $\Omega(\%)$ & $N^{*}$ & $T^{*}$ & $\Psi(\%)$ & $C_{\mathcal{A}}$ & $\Omega(\%)$ & $N^{*}$ & $T^{*}$ & $\Psi(\%)$ & $C_{\mathcal{A}}$ & $\Omega(\%)$ \\
\hline 0.1 & 15 & 8 & 5 & 0.350 & 0.14 & 1 & 9.1 & 11. & 0.352 & 0.6 & 1 & 11 & 35.80 & 0.362 & 3.43 \\
\hline 0.5 & 13 & 10.43 & 5.04 & 0.391 & 0.10 & 13 & 10.92 & 9.97 & 0.392 & 0.44 & 13 & 12.38 & 24.67 & 0.401 & 2.66 \\
\hline 1 & 12 & 11.51 & 4.45 & 0.435 & 0.09 & 12 & 12.01 & 8.98 & 0.436 & 0.35 & 11 & 14.16 & 28.49 & 0.443 & 2.10 \\
\hline 2 & 10 & 13.71 & 3.79 & 0.509 & 0.06 & 10 & 14.20 & 7.49 & 0.510 & 0.24 & 10 & 15.67 & 18.62 & 0.516 & 1.49 \\
\hline 5 & 8 & 17.12 & 2.95 & 0.684 & 0.03 & 7 & 18.53 & 11.43 & 0.685 & 0.13 & 7 & 20.01 & 20.32 & 0.690 & 0.85 \\
\hline
\end{tabular}
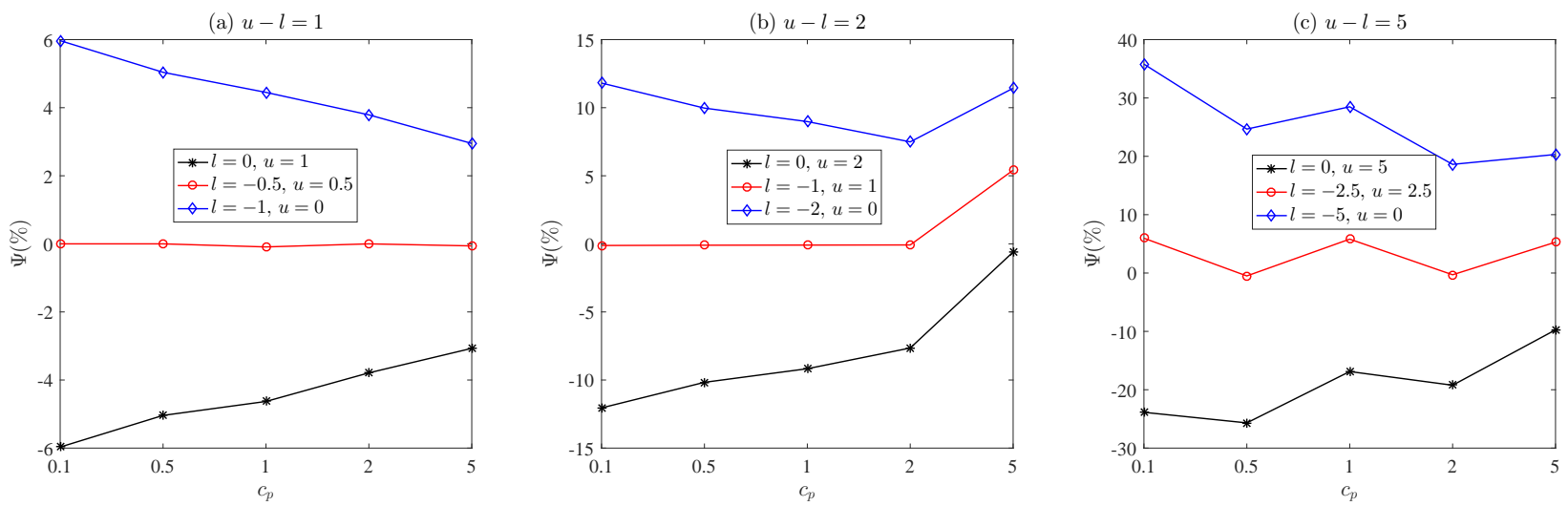

Fig. 2. Relative PM-Interval Change $\Psi$ in The Infinite Horizon Setting $(\beta=3.5)$.

absolute value of $\Psi$ ) tends to become larger when $u-l$ increases and/or $c_{p}$ decreases.

(4) In terms of average cost rates, we find that $C_{\mathcal{A}}\left(N^{*}, T^{*}\right)$ and $\widetilde{C}_{\mathcal{A}}\left(\widetilde{N}^{*}, \widetilde{T}^{*}\right)$ are identical for $\beta=1.5$; while $C_{\mathcal{A}}\left(N^{*}, T^{*}\right)$ is slightly larger than $\widetilde{C}_{\mathcal{A}}\left(\widetilde{N}^{*}, \widetilde{T}^{*}\right)$ in the case of $\beta=3.5$. One thing noteworthy is that when $\beta=3.5$, the average cost rate $C_{\mathcal{A}}\left(N^{*}, T^{*}\right)$ remains constant for the same value of $u-l$, irrespective of the unpunctuality scenarios (see Table III).

\section{Model B: Finite Planning-Horizon Setting}

In this section, we investigate an unpunctual imperfect PM policy for a finite planning horizon (termed "Model B"). We generalize the periodic imperfect PM model in Nakagawa and Mizutani [16] by assuming that the PM deviations are governed by a specific probability distribution. Below we first formulate the total expected maintenance costs for the punctual and unpunctual execution scenarios, respectively, and then discuss the optimization of the two scenarios. Numerical experiments are also performed to illustrate the proposed PM models.

\section{A. The Optimization Models}

For the imperfect PM policy in a finite horizon, the assumptions (i), (iii)-(vi) in Section II-A still hold; while the assumption (ii) is revised as follows: The planning horizon is $[0, S]$, and the $j$ th PM action is carried out at instant $j T+Y_{j}$, $j=1,2, \ldots, N$, with $S=(N+1) T$; see Fig. 3. It should be noted that there is no imperfect PM or replacement at the end of the planning horizon. This setting is appropriate for a relatively short planning horizon (e.g., warranty period, lease period) in the sense that the maintainer is responsible for maintenance execution only in $[0, S]$ and the system will continue to operate after $S$.

Remark 4: Due to the infinite/finite nature of the planning horizons, the instants of performing the $j$ th PM action are 


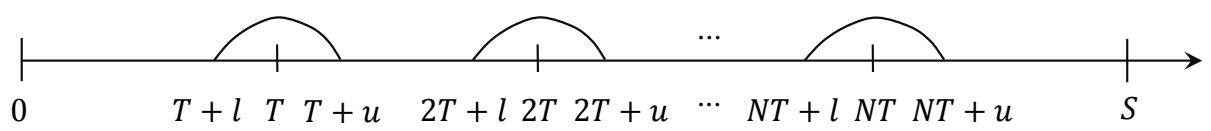

Fig. 3. Illustration of The PM Schedule Within a Finite Planning Horizon $(l \leq 0 \leq u)$.

indeed different in the two settings. Specifically, the $j$ th PM instant in the infinite horizon setting is $j T+\sum_{i=1}^{j} Y_{i}$, whereas that in the finite horizon setting is $j T+Y_{j}$. In the infinite horizon setting, the PM deviation $Y_{j}$ is applied to the $j$ th PM interval, thus it will accumulate for subsequent PM instants. However, $Y_{j}$ is applied to the $j$ th PM instant directly in the finite horizon setting, so it has no impact on subsequent PM instants. This is because in the finite horizon setting, the planning horizon ends at $S$ so that the $N$ th PM instant shall be smaller than $S$; whereas the infinite horizon setting does not need this constraint.

Based on the aforementioned assumptions, if all PM actions are always executed on time, then the total expected maintenance cost in $[0, S]$ is

$$
\widetilde{C}_{\mathcal{B}}(N)=c_{m} \sum_{j=1}^{N+1} A_{j-1} \Lambda(T)+N c_{p} .
$$

The objective is to determine the optimal number of PM actions $\widetilde{N}^{*}$ such that $\widetilde{C}_{\mathcal{B}}(N)$ is minimized. Once the optimal number of PM actions $\widetilde{N}^{*}$ is identified, the optimal PM interval can be determined as $\widetilde{T}^{*}=S /\left(\widetilde{N}^{*}+1\right)$.

On the other hand, in the unpunctual execution scenario (see Fig. 3), the $j$ th PM instant is $j T+Y_{j}$, and the $j$ th PM interval is thus $j T+Y_{j}-\left((j-1) T+Y_{j-1}\right)=T+Y_{j}-Y_{j-1}$, $j=2,3, \ldots, N$. Notice that the first and the last PM intervals are $T+Y_{1}$ and $T-Y_{N}$, respectively. Then, the total expected maintenance cost in $[0, S]$ can be derived as

$$
\begin{aligned}
C_{\mathcal{B}}(N)= & c_{m} \int_{l}^{u} \Lambda\left(T+y_{1}\right) \mathrm{d} G\left(y_{1}\right)+c_{m} \sum_{j=2}^{N} \int_{l}^{u} \int_{l}^{u} A_{j-1} \\
& \times \Lambda\left(T+y_{j}-y_{j-1}\right) \mathrm{d} G\left(y_{j-1}\right) \mathrm{d} G\left(y_{j}\right) \\
& +c_{m} \int_{l}^{u} A_{N} \Lambda\left(T-y_{N}\right) \mathrm{d} G\left(y_{N}\right)+N c_{p}
\end{aligned}
$$

Likewise, the optimization problem is to seek the optimal $N^{*}$ to minimize $C_{\mathcal{B}}(N)$, when the maintenance unpunctuality is anticipated.

One thing noteworthy is that in order to avoid impractical PM plans, each PM interval should be larger than zero, i.e., the $j$ th PM instant should be larger than the $(j-1)$ th instant (see Fig. 3). Mathematically, the condition is equivalent to $T+Y_{j}-Y_{j-1}>0, j=2,3, \ldots, N$. Considering the range of $Y_{j}$ and $Y_{j-1}$, i.e., $[l, u]$, we have $T>u-l$. This means that the PM interval $T$ should be larger than the unpunctual range $u-l$. As a result, the optimal number of PM action $N^{*}$ has an upper bound: $N^{*} \leq N_{\max }=\lfloor S /(u-l)-1\rfloor$, where $\lfloor x\rfloor$ represents the largest integer that is small than $x$.

Remark 5: When $N=0$, i.e., no imperfect PM action is carried out within $[0, S]$, the total expected maintenance costs in (12) and (13) reduce to $\widetilde{C}_{\mathcal{B}}(N=0)=C_{\mathcal{B}}(N=0)=$ $c_{m} \Lambda(S)$.

\section{B. Optimal PM Decisions}

In this subsection, we examine the optimal finite-horizon PM decisions for the punctual and unpunctual scenarios, respectively.

We first look at the optimal number of PM actions $N^{*}$ for the unpunctual execution scenario. By forming inequalities $C_{\mathcal{B}}(N-1)>C_{\mathcal{B}}(N)$ and $C_{\mathcal{B}}(N) \leq C_{\mathcal{B}}(N+1)$, we have

$$
\mathcal{J}_{\mathcal{B}}(N+1) \leq \frac{c_{p}}{c_{m}} \text { and } \mathcal{J}_{\mathcal{B}}(N)>\frac{c_{p}}{c_{m}}
$$

where

$$
\begin{aligned}
\mathcal{J}_{\mathcal{B}}(N)= & \int_{l}^{u} \Lambda\left(\frac{S}{N}+y_{1}\right) \mathrm{d} G\left(y_{1}\right)+\sum_{j=2}^{N-1} \int_{l}^{u} \int_{l}^{u} A_{j-1} \\
& \times \Lambda\left(\frac{S}{N}+y_{j}-y_{j-1}\right) \mathrm{d} G\left(y_{j-1}\right) \mathrm{d} G\left(y_{j}\right) \\
& +\int_{l}^{u} A_{N-1} \Lambda\left(\frac{S}{N}-y_{N}\right) \mathrm{d} G\left(y_{N}\right) \\
& -\int_{l}^{u} \Lambda\left(\frac{S}{N+1}+y_{1}\right) \mathrm{d} G\left(y_{1}\right)-\sum_{j=2}^{N} \int_{l}^{u} \int_{l}^{u} \\
& A_{j-1} \Lambda\left(\frac{S}{N+1}+y_{j}-y_{j-1}\right) \mathrm{d} G\left(y_{j-1}\right) \mathrm{d} G\left(y_{j}\right) \\
& -\int_{l}^{u} A_{N} \Lambda\left(\frac{S}{N+1}-y_{N+1}\right) \mathrm{d} G\left(y_{N+1}\right) .
\end{aligned}
$$

Likewise, for the finite-horizon PM policy with punctual executions, the optimal $\widetilde{N}^{*}$ can be determined by

$$
\widetilde{\mathcal{J}}_{\mathcal{B}}(N+1) \leq \frac{c_{p}}{c_{m}} \text { and } \widetilde{\mathcal{J}}_{\mathcal{B}}(N)>\frac{c_{p}}{c_{m}}
$$

where

$$
\widetilde{\mathcal{J}}_{\mathcal{B}}(N)=\sum_{j=1}^{N} A_{j-1} \Lambda\left(\frac{S}{N}\right)-\sum_{j=1}^{N+1} A_{j-1} \Lambda\left(\frac{S}{N+1}\right) .
$$

Note that it is difficult, if not impossible, to show the uniqueness of optimal $N^{*}$ and $\widetilde{N}^{*}$ for the unpunctual and punctual execution scenarios. Fortunately, both $N^{*}$ and $\widetilde{N}^{*}$ are integers, so simple search methods are efficient enough for obtaining them. Therefore, the impact of unpunctual policy executions on the optimal PM decisions in the finite horizon setting will be investigated via numerical experiments. 

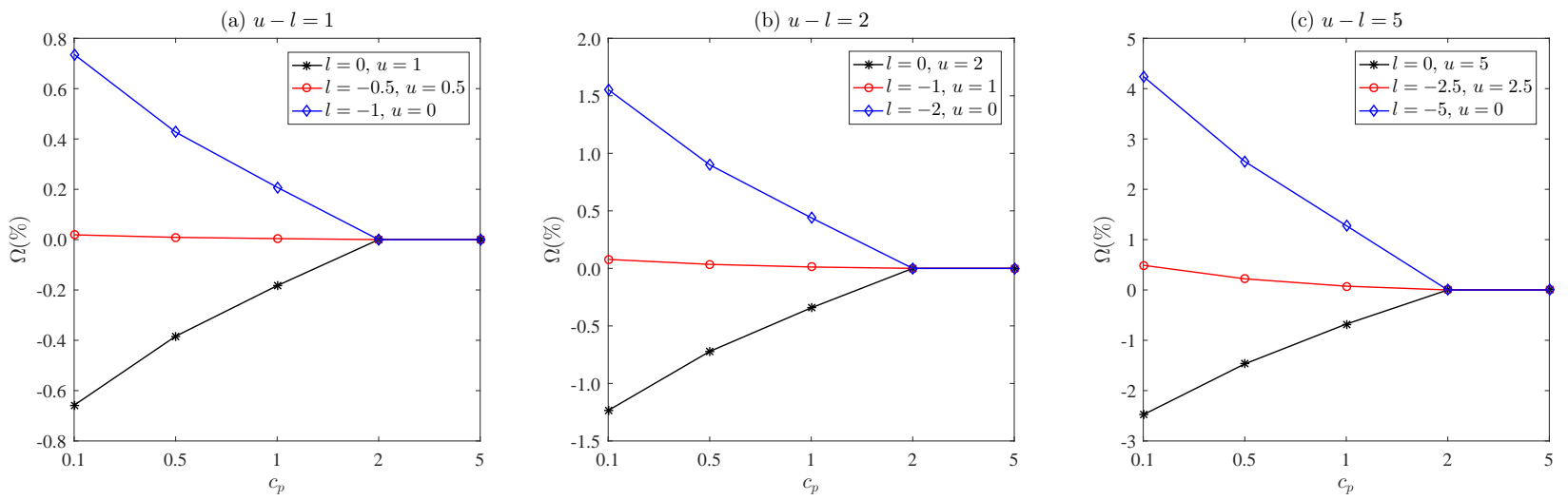

Fig. 4. Relative Maintenance-Cost Change $\Omega$ in The Finite Horizon Setting $(\beta=1.5)$.

TABLE IV

Optimal PM Decisions Under Punctual Executions in The Finite HORIZON SETTING.

\begin{tabular}{lcclcc}
\hline & \multicolumn{2}{c}{$\beta=1.5$} & & \multicolumn{2}{c}{$\beta=3.5$} \\
\cline { 2 - 3 } \cline { 5 - 6 }$c_{p}$ & $\widetilde{N}^{*}$ & $\widetilde{C}_{\mathcal{B}}$ & & $\widetilde{N}^{*}$ & $\widetilde{C}_{\mathcal{B}}$ \\
\hline 0.1 & 3 & 5.772 & & 10 & 2.106 \\
0.5 & 2 & 6.645 & & 6 & 4.959 \\
1 & 1 & 7.223 & & 4 & 7.527 \\
2 & 0 & 8.000 & & 3 & 11.472 \\
5 & 0 & 8.000 & & 2 & 20.036 \\
\hline
\end{tabular}

\section{Numerical Studies}

In this subsection, numerical experiments are conducted to illustrate the unpunctual PM policy for a finite planning horizon, and to examine the impact of unpunctual executions on the optimal PM decisions. The parameter setting is the same as that in Section II-D. That is, we adopt the same setting with respect to hazard rate function and unpunctuality scenarios and magnitudes. In addition, the length of the finite planning horizon is set to $S=60$.

We first examine the imperfect PM policy in the punctual execution scenario. Table IV lists the optimal number of PM actions and corresponding total maintenance cost for different values of $c_{p}$. It can be observed that as the PM cost $c_{p}$ increases, the optimal number of PM actions $\widetilde{N}^{*}$ decreases, whereas the total maintenance cost $\widetilde{C}_{\mathcal{B}}\left(\widetilde{N}^{*}\right)$ increases. This finding applies to both concave and convex hazard rates.

We then look at the imperfect PM policy in the unpunctual execution scenario. Tables V and VI summarize the optimal PM decisions and corresponding total maintenance costs under unpunctual executions for $\beta=1.5$ and $\beta=3.5$, respectively. Figs. 4 and 5 further show the values of relative maintenance cost changes $\Omega$ for $\beta=1.5$ and $\beta=3.5$, respectively. From these tables and figures, the following findings can be drawn:

(1) Analogous to the observation in the punctual execution scenario, as the PM cost $c_{p}$ increases, the optimal number of PM actions $N^{*}$ decreases, whereas the total expected maintenance cost $C_{\mathcal{B}}\left(N^{*}\right)$ increases. This finding applies to all numerical cases in Tables V and VI, irrespective of the unpunctuality scenarios and magnitudes.

(2) Compared with the punctual execution scenario, the unpunctual PM policy requires the same, or slightly fewer (especially when the unpunctuality magnitude, $u-l$, is large) number of PM actions for $\beta=1.5$ and $\beta=3.5$. It shows that the difference between $N^{*}$ and $\widetilde{N}^{*}$ depends more on the unpunctuality magnitude, rather than the unpunctuality scenario.

(3) Different from the infinite horizon setting, it is interesting to find that the total maintenance cost of the unpunctual PM policy can be either higher or lower than that of the punctual policy, depending on the unpunctuality scenario and magnitude (see Figs. 4 and 5). More specifically, (i) when $0 \leq l<u, C_{\mathcal{B}}\left(N^{*}\right)<\widetilde{C}_{\mathcal{B}}\left(\widetilde{N}^{*}\right)$ in most cases $\left(C_{\mathcal{B}}\left(N^{*}\right)>\widetilde{C}_{\mathcal{B}}\left(\widetilde{N}^{*}\right)\right.$ only when $\beta=3.5$ and $u-l=5$ ); (ii) when $l<u \leq 0, C_{\mathcal{B}}\left(N^{*}\right)>\widetilde{C}_{\mathcal{B}}\left(\widetilde{N}^{*}\right)$; while (iii) when $l<0<u, C_{\mathcal{B}}\left(N^{*}\right)$ is slightly higher than $\widetilde{C}_{\mathcal{B}}\left(\widetilde{N}^{*}\right)$, especially when $u-l$ is not large. Moreover, the difference between $C_{\mathcal{B}}\left(N^{*}\right)$ and $\widetilde{C}_{\mathcal{B}}\left(\widetilde{N}^{*}\right)$ (measured by the absolute value of $\Omega$ ) tends to become larger when $u-l$ increases and/or $c_{p}$ decreases.

\section{CONCLUSION}

In this article, we have studied two imperfect PM policies with unpunctual executions for infinite and finite planning horizons, respectively. The two PM models extend their punctual counterparts by taking into account the unpunctual executions of scheduled PM activities. From the mathematical modeling perspective, the maintenance unpunctuality is assumed to be governed by a specific probability distribution. We have discussed the modeling and optimization of the two unpunctual PM policies, and then explored the impact of unpunctual executions on the optimal PM decisions and corresponding maintenance expenses in an analytical or numerical way.

Numerical experiments show that the impacts of unpunctual executions on the optimal PM decisions and maintenance costs (or cost rates), in the infinite and finite horizon settings, are indeed different. In the infinite horizon setting, the optimal PM interval in the unpunctual execution scenario can be longer or shorter than that in the punctual scenario, depending on the 
TABLE V

Optimal PM Decisions Under Unpunctual Executions in The Finite Horizon Setting $(\beta=1.5)$.

\begin{tabular}{|c|c|c|c|c|c|c|c|c|c|}
\hline \multirow[b]{2}{*}{$c_{p}$} & \multicolumn{3}{|c|}{$l=0, u=1$} & \multicolumn{3}{|c|}{$l=0, u=2$} & \multicolumn{3}{|c|}{$l=0, u=5$} \\
\hline & $N^{*}$ & $C_{\mathcal{B}}$ & $\Omega(\%)$ & $N^{*}$ & $C_{\mathcal{B}}$ & $\Omega(\%)$ & $N^{*}$ & $C_{\mathcal{B}}$ & $\Omega(\%)$ \\
\hline 0.1 & 3 & 5.734 & -0.66 & 3 & 5.701 & -1.24 & 3 & 5.629 & -2.48 \\
\hline 0.5 & 2 & 6.620 & -0.38 & 2 & 6.597 & -0.72 & 2 & 6.548 & -1.47 \\
\hline 1 & 1 & 7.209 & -0.18 & 1 & 7.198 & -0.34 & 1 & 7.174 & -0.68 \\
\hline 2 & 0 & 8.000 & 0.00 & 0 & 8.000 & 0.00 & 0 & 8.000 & 0.00 \\
\hline \multirow[t]{2}{*}{5} & 0 & 8.000 & 0.00 & 0 & 8.000 & 0.00 & 0 & 8.000 & 0.00 \\
\hline & \multicolumn{3}{|c|}{$l=-0.5, u=0.5$} & \multicolumn{3}{|c|}{$l=-1, u=1$} & \multicolumn{3}{|c|}{$l=-2.5, u=2.5$} \\
\hline$c_{p}$ & $N^{*}$ & $C_{\mathcal{B}}$ & $\Omega(\%)$ & $N^{*}$ & $C_{\mathcal{B}}$ & $\Omega(\%)$ & $N^{*}$ & $C_{\mathcal{B}}$ & $\Omega(\%)$ \\
\hline 0.1 & 3 & 5.773 & 0.02 & 3 & 5.776 & 0.08 & 3 & 5.800 & 0.49 \\
\hline 0.5 & 2 & 6.646 & 0.01 & 2 & 6.648 & 0.03 & 2 & 6.660 & 0.22 \\
\hline 1 & 1 & 7.223 & 0.00 & 1 & 7.223 & 0.01 & 1 & 7.228 & 0.07 \\
\hline 2 & 0 & 8.000 & 0.00 & 0 & 8.000 & 0.00 & 0 & 8.000 & 0.00 \\
\hline \multirow[t]{2}{*}{5} & 0 & 8.000 & 0.00 & 0 & 8.000 & 0.00 & 0 & 8.000 & 0.00 \\
\hline & \multicolumn{3}{|c|}{$l=-1, u=0$} & \multicolumn{3}{|c|}{$l=-2, u=0$} & \multicolumn{3}{|c|}{$l=-5, u=0$} \\
\hline$c_{p}$ & $N^{*}$ & $C_{\mathcal{B}}$ & $\Omega(\%)$ & $N^{*}$ & $C_{\mathcal{B}}$ & $\Omega(\%)$ & $N^{*}$ & $C_{\mathcal{B}}$ & $\Omega(\%)$ \\
\hline 0.1 & 3 & 5.814 & 0.74 & 3 & 5.862 & 1.55 & 2 & 6.017 & 4.24 \\
\hline 0.5 & 2 & 6.674 & 0.43 & 2 & 6.705 & 0.90 & 1 & 6.815 & 2.55 \\
\hline 1 & 1 & 7.238 & 0.21 & 1 & 7.254 & 0.44 & 1 & 7.315 & 1.28 \\
\hline 2 & 0 & 8.000 & 0.00 & 0 & 8.000 & 0.00 & 0 & 8.000 & 0.00 \\
\hline 5 & 0 & 8.000 & 0.00 & 0 & 8.000 & 0.00 & 0 & 8.000 & 0.00 \\
\hline
\end{tabular}

TABLE VI

Optimal PM Decisions Under Unpunctual Executions in The Finite Horizon Setting $(\beta=3.5)$.

\begin{tabular}{|c|c|c|c|c|c|c|c|c|c|}
\hline \multirow[b]{2}{*}{$c_{p}$} & \multicolumn{3}{|c|}{$l=0, u=1$} & \multicolumn{3}{|c|}{$l=0, u=2$} & \multicolumn{3}{|c|}{$l=0, u=5$} \\
\hline & $N^{*}$ & $C_{\mathcal{B}}$ & $\Omega(\%)$ & $N^{*}$ & $C_{\mathcal{B}}$ & $\Omega(\%)$ & $N^{*}$ & $C_{\mathcal{B}}$ & $\Omega(\%)$ \\
\hline 0.1 & 10 & 2.072 & -1.60 & 9 & 2.101 & -0.23 & 9 & 2.500 & 18.70 \\
\hline 0.5 & 6 & 4.914 & -0.90 & 6 & 4.919 & -0.79 & 6 & 5.220 & 5.28 \\
\hline 1 & 4 & 7.470 & -0.76 & 4 & 7.463 & -0.85 & 4 & 7.731 & 2.72 \\
\hline 2 & 3 & 11.405 & -0.59 & 3 & 11.390 & -0.71 & 3 & 11.659 & 1.63 \\
\hline \multirow[t]{2}{*}{5} & 2 & 19.955 & -0.41 & 2 & 19.934 & -0.51 & 2 & 20.233 & 0.98 \\
\hline & \multicolumn{3}{|c|}{$l=-0.5, u=0.5$} & \multicolumn{3}{|c|}{$l=-1, u=1$} & \multicolumn{3}{|c|}{$l=-2.5, u=2.5$} \\
\hline$c_{p}$ & $N^{*}$ & $C_{\mathcal{B}}$ & $\Omega(\%)$ & $N^{*}$ & $C_{\mathcal{B}}$ & $\Omega(\%)$ & $N^{*}$ & $C_{\mathcal{B}}$ & $\Omega(\%)$ \\
\hline 0.1 & 10 & 2.130 & 1.13 & 9 & 2.200 & 4.44 & 9 & 2.659 & 26.26 \\
\hline 0.5 & 6 & 4.975 & 0.33 & 6 & 5.024 & 1.32 & 6 & 5.370 & 8.30 \\
\hline 1 & 4 & 7.541 & 0.19 & 4 & 7.583 & 0.75 & 4 & 7.881 & 4.71 \\
\hline 2 & 3 & 11.485 & 0.12 & 3 & 11.525 & 0.46 & 3 & 11.802 & 2.88 \\
\hline \multirow[t]{2}{*}{5} & 2 & 20.048 & 0.06 & 2 & 20.085 & 0.24 & 2 & 20.340 & 1.52 \\
\hline & \multicolumn{3}{|c|}{$l=-1, u=0$} & \multicolumn{3}{|c|}{$l=-2, u=0$} & \multicolumn{3}{|c|}{$l=-5, u=0$} \\
\hline$c_{p}$ & $N^{*}$ & $C_{\mathcal{B}}$ & $\Omega(\%)$ & $N^{*}$ & $C_{\mathcal{B}}$ & $\Omega(\%)$ & $N^{*}$ & $C_{\mathcal{B}}$ & $\Omega(\%)$ \\
\hline 0.1 & 10 & 2.207 & 4.80 & 9 & 2.373 & 12.68 & 8 & 3.282 & 55.83 \\
\hline 0.5 & 6 & 5.054 & 1.93 & 6 & 5.203 & 4.93 & 6 & 5.992 & 20.84 \\
\hline 1 & 4 & 7.631 & 1.38 & 4 & 7.783 & 3.40 & 4 & 8.565 & 13.79 \\
\hline 2 & 3 & 11.593 & 1.05 & 3 & 11.768 & 2.58 & 3 & 12.597 & 9.81 \\
\hline 5 & 2 & 20.178 & 0.71 & 2 & 20.383 & 1.73 & 2 & 21.372 & 6.67 \\
\hline
\end{tabular}

unpunctuality scenario and magnitude; however, the resultant cost rate is always higher than or equal to that in the punctual scenario. On the other hand, in the finite horizon setting, the total maintenance cost in the unpunctual scenario can be higher or lower than that in the punctual scenario, depending on the unpunctuality scenario and magnitude as well. These insights are helpful for maintenance planners to adjust their PM schedules according to the planning horizons of interest, when the maintenance unpunctuality is well anticipated.

It is worth emphasizing that we have confined our analysis of the unpunctual PM policies to numerical experiments, as the complexity of the cost (rate) models makes it quite difficult to obtain analytical results. As a consequence, we have been cautious about making general claims about the impacts of unpunctual executions. We recognize this as a limitation of the work. Deriving general analytical results on the comparison of punctual and unpunctual scenarios is thus valuable, and needs in-depth investigations. Furthermore, a consideration of practical significance is that the unpunctual ranges might become wider or narrower when time elapsed, rather than 

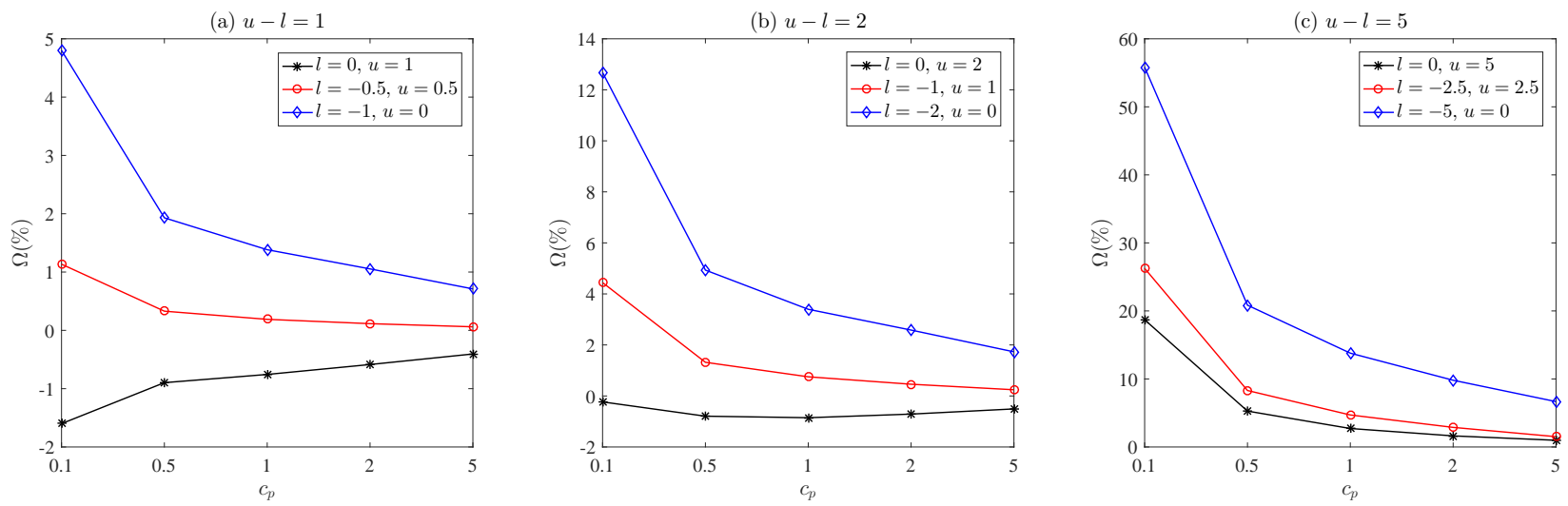

Fig. 5. Relative Maintenance-Cost Change $\Omega$ in The Finite Horizon Setting ( $\beta=3.5$ ).

remaining unchanged. A potential way of addressing this issue is to model the PM deviation as a stochastic process with an increasing or decreasing mean in $N$ and $T$. In addition, future research could also extend the current framework to other imperfect PM models, e.g., age reduction model, hybrid model, and geometric process model, or more advanced maintenance paradigms, e.g., condition-based maintenance and predictive maintenance.

\section{APPENDIX}

\section{A. Proof of Proposition 1}

It is evident that $\mathcal{J}_{\mathcal{A}}(T, N)$ is increasing in $N$ and approaches to $\infty$ as $N \rightarrow \infty$, because

$$
\begin{aligned}
& \mathcal{J}_{\mathcal{A}}(T, N)-\mathcal{J}_{\mathcal{A}}(T, N-1) \\
& =N\left(A_{N}-A_{N-1}\right) \int_{l}^{u} \Lambda\left(T+y_{N}\right) \mathrm{d} G\left(y_{N}\right)>0,
\end{aligned}
$$

and

$$
\begin{aligned}
& \lim _{N \rightarrow \infty} \mathcal{J}_{\mathcal{A}}(T, N) \\
& =\lim _{N \rightarrow \infty}\left(\int_{l}^{u} \Lambda(T+y) \mathrm{d} G(y)\right) \sum_{j=1}^{N}\left(A_{N}-A_{j-1}\right)=\infty .
\end{aligned}
$$

The last equality is due to $a_{j}>1, j=1,2, \ldots, N-1$, and thus $\lim _{N \rightarrow \infty} \sum_{j=1}^{N}\left(A_{N}-A_{j-1}\right)=\infty$. Therefore, there exists a finite and unique $N^{*}$ that minimizes $C_{\mathcal{A}}(T, N)$ for any feasible $T>\max \{-l, 0\}$.

\section{B. Proof of Proposition 2}

As the hazard rate $\lambda(t)$ is an increasing function of $t$, i.e., $\lambda^{\prime}(t)>0$, we have

$$
\mathcal{L}_{\mathcal{A}, i}^{\prime}(T)=\left(T+\mu_{y}\right) \int_{l}^{u} A_{j-1} \lambda^{\prime}\left(T+y_{j}\right) \mathrm{d} G\left(y_{j}\right)>0 .
$$

Following the same argument as in Barlow and Proschan [40, p. 97] and $\mathrm{He}$ et al. [38], we show that $\mathcal{L}_{\mathcal{A}, i}(T) \rightarrow \infty$ as $T \rightarrow \infty$. First, it is clear that $\mathcal{L}_{\mathcal{A}, i}(T)$ is continuous on $[0,+\infty)$. Let $\left\{T_{i}\right\}$ be an infinite sequence such that $\max \left\{-\mu_{y}, 0\right\}<T_{1}<T_{2}<\ldots$, with $\lambda\left(T_{i-1}\right)<\lambda\left(T_{i}\right)$ for $i=2,3, \ldots$ and $\lim _{i \rightarrow \infty} \lambda\left(T_{i}\right)=\infty$. Such a sequence exists as $\lambda(t)$ is strictly increasing and unbounded (by Assumption (i)). Then, it is evident that

$$
\begin{aligned}
\mathcal{L}_{\mathcal{A}, i}\left(T_{i}\right)= & \left(T_{i}+\mu_{y}\right) \int_{l}^{u} A_{j-1} \lambda\left(T_{i}+y_{j}\right) \mathrm{d} G\left(y_{j}\right) \\
& -\int_{l}^{u} A_{j-1} \Lambda\left(T_{i}+y_{j}\right) \mathrm{d} G\left(y_{j}\right) \\
> & \left(T_{1}+\mu_{y}\right) \int_{l}^{u} A_{j-1} \lambda\left(T_{i}+y_{j}\right) \mathrm{d} G\left(y_{j}\right) \\
& -\int_{l}^{u} A_{j-1} \Lambda\left(T_{1}+y_{j}\right) \mathrm{d} G\left(y_{j}\right) \\
\rightarrow & \infty \text { as } i \rightarrow \infty .
\end{aligned}
$$

In other words, $\mathcal{L}_{\mathcal{A}, i}(T)$ is increasing in $T$ and tends to $\infty$ as $T \rightarrow \infty$.

Therefore, the solution to (4) is unique and finite when condition (5) is met; otherwise, $\inf C_{\mathcal{A}}(T, N)=$ $\lim _{T \rightarrow \max \{-l, 0\}} C_{\mathcal{A}}(T, N)$. Given the optimal solution $T^{*}$ to (4), rearranging its terms yields (6).

\section{Proof of Theorem 1}

Part (i): Recall that both $\mathcal{L}_{\mathcal{A}, i}(T)$ and $\widetilde{\mathcal{L}}_{\mathcal{A}, i}(T)$ are increasing functions of $T$. Also, $0 \leq l<u$ implies that $\mu_{y}>0$ and both problems have the same feasible domain $\{T \mid T>0\}$. According to (4) and (9), if $\mathcal{L}_{\mathcal{A}, i}(T)>\widetilde{\mathcal{L}}_{\mathcal{A}, i}(T)$ is true for all $T>0$, then $T^{*}<\widetilde{T}^{*}$ for a fixed $N$.

First, it is clear that $\lim _{T \rightarrow 0} \widetilde{\mathcal{L}}_{\mathcal{A}, i}(T)=0$ as $\lambda(0)=0$. Hence, if condition (11) is satisfied, then we have

$$
\lim _{T \rightarrow 0} \mathcal{L}_{\mathcal{A}, i}(T) \geq \lim _{T \rightarrow 0} \widetilde{\mathcal{L}}_{\mathcal{A}, i}(T) .
$$

Next, $\mathcal{L}_{\mathcal{A}, i}(T)$ is increasing faster than $\widetilde{\mathcal{L}}_{\mathcal{A}, i}(T)$, since

$$
\begin{aligned}
\mathcal{L}_{\mathcal{A}, i}^{\prime}(T) & =\left(T+\mu_{y}\right) \int_{l}^{u} A_{j-1} \lambda^{\prime}\left(T+y_{j}\right) \mathrm{d} G\left(y_{j}\right) \\
& >T \int_{l}^{u} A_{j-1} \lambda^{\prime}(T) \mathrm{d} G\left(y_{j}\right) \\
& =T A_{j-1} \lambda^{\prime}(T)=\widetilde{\mathcal{L}}_{\mathcal{A}, i}^{\prime}(T) .
\end{aligned}
$$

The inequality is due to $0 \leq l<u\left(\mu_{y}>0\right)$ and the convexity of $\lambda(t)$. Hence, it is true that $\mathcal{L}_{\mathcal{A}, i}(T)>\widetilde{\mathcal{L}}_{\mathcal{A}, i}(T)$ for all $T>0$. If the two policies adopt the same $N \geq 1$, then 
$\sum_{j=1}^{N} \mathcal{L}_{\mathcal{A}, i}(T)>\sum_{j=1}^{N} \widetilde{\mathcal{L}}_{\mathcal{A}, i}(T)$ for all $T>0$, which implies $T^{*}<\widetilde{T}^{*}$.

Part (ii): Analogously, notice that both $\mathcal{J}_{\mathcal{A}}(T, N)$ and $\widetilde{\mathcal{J}}_{\mathcal{A}}(T, N)$ are increasing functions of $N$. If we can show that $\mathcal{J}_{\mathcal{A}}(T, N)>\widetilde{\mathcal{J}}_{\mathcal{A}}(T, N)$ for all $N \geq 1$, then $N^{*} \leq \widetilde{N}^{*}$ follows directly, according to (3) and (8). The inequality is not strict because $N$ should be integer, thus there is a possibility that $N^{*}=\widetilde{N}^{*}$.

Based on Jensen's inequality, we know that

$$
\begin{aligned}
& \mathcal{J}_{\mathcal{A}}(T, N)-\mathcal{J}_{\mathcal{A}}(T, N-1) \\
& =N\left(A_{N}-A_{N-1}\right) \int_{l}^{u} \Lambda\left(T+y_{N}\right) \mathrm{d} G\left(y_{N}\right) \\
& \geq N\left(A_{N}-A_{N-1}\right) \Lambda\left(T+\mu_{y}\right) \\
& >N\left(A_{N}-A_{N-1}\right) \Lambda(T) \\
& =\widetilde{\mathcal{J}}_{\mathcal{A}}(T, N)-\widetilde{\mathcal{J}}_{\mathcal{A}}(T, N-1) .
\end{aligned}
$$

Thus, $\mathcal{J}_{\mathcal{A}}(T, N)$ indeed increases faster than $\widetilde{\mathcal{J}}_{\mathcal{A}}(T, N)$. Combining with the fact $\mathcal{J}_{\mathcal{A}}(T, 0)=\widetilde{\mathcal{J}}_{\mathcal{A}}(T, 0)=0$, we conclude that $\mathcal{J}_{\mathcal{A}}(T, N)>\widetilde{\mathcal{J}}_{\mathcal{A}}(T, N)$ for all $N \geq 1$. Therefore, if the two policies specify the same $T$, we have $N^{*} \leq \widetilde{N}^{*}$.

\section{Proof of Theorem 2}

Part (i): In this case, $l<u \leq 0$ implies that $\mu_{y}<0$ and the feasible domain of $T$ in the unpunctual scenario is $\{T \mid T>$ $-l\}$. Likewise, we first show $\lim _{T \rightarrow-l} \mathcal{L}_{\mathcal{A}, i}(T)<\widetilde{\mathcal{L}}_{\mathcal{A}, i}(-l)$, and then show $\mathcal{L}_{\mathcal{A}, i}(T)$ is increasing slower than $\widetilde{\mathcal{L}}_{\mathcal{A}, i}(T)$.

The expressions of $\lim _{T \rightarrow-l} \mathcal{L}_{\mathcal{A}, i}(T)$ and $\widetilde{\mathcal{L}}_{\mathcal{A}, i}(-l)$ are given by

$$
\begin{aligned}
\lim _{T \rightarrow-l} \mathcal{L}_{\mathcal{A}, i}(T)= & \left(-l+\mu_{y}\right) \int_{l}^{u} A_{j-1} \lambda\left(-l+y_{j}\right) \mathrm{d} G\left(y_{j}\right) \\
& -\int_{l}^{u} A_{j-1} \Lambda\left(-l+y_{j}\right) \mathrm{d} G\left(y_{j}\right),
\end{aligned}
$$

and

$$
\begin{aligned}
\widetilde{\mathcal{L}}_{\mathcal{A}, i}(-l) & =-l A_{j-1} \lambda(-l)-A_{j-1} \Lambda(-l) \\
& =\left(-l+\mu_{y}\right) A_{j-1} \lambda(-l)-A_{j-1}\left[\Lambda(-l)+\mu_{y} \lambda(-l)\right] .
\end{aligned}
$$

Notice that $\int_{l}^{u} \lambda\left(-l+y_{j}\right) \mathrm{d} G\left(y_{j}\right)<\lambda(-l)$ for $l<u \leq 0$. According to the Jensen's inequality (and bear in mind that $\Lambda(t)$ is convex), we obtain

$$
\begin{aligned}
\int_{l}^{u} \Lambda\left(-l+y_{j}\right) \mathrm{d} G\left(y_{j}\right) & \geq \Lambda\left(-l+\mu_{y}\right) \\
& =\Lambda(-l)-\int_{-l+\mu_{y}}^{-l} \lambda(t) \mathrm{d} t .
\end{aligned}
$$

As $\lambda(t)$ is convex, we also have

$$
-\int_{-l+\mu_{y}}^{-l} \lambda(t) \mathrm{d} t \geq-\lambda(-l)\left(-\mu_{y}\right)=\lambda(-l) \mu_{y} .
$$

The discussions above lead to the result: $\lim _{T \rightarrow-l} \mathcal{L}_{\mathcal{A}, i}(T)<$ $\widetilde{\mathcal{L}}_{\mathcal{A}, i}(-l)$.
We claim that $\mathcal{L}_{\mathcal{A}, i}(T)$ is increasing slower than $\widetilde{\mathcal{L}}_{\mathcal{A}, i}(T)$ for $l<u \leq 0$, as

$$
\begin{aligned}
\mathcal{L}_{\mathcal{A}, i}^{\prime}(T) & =\left(T+\mu_{y}\right) \int_{l}^{u} A_{j-1} \lambda^{\prime}\left(T+y_{j}\right) \mathrm{d} G\left(y_{j}\right) \\
& <T \int_{l}^{u} A_{j-1} \lambda^{\prime}(T) \mathrm{d} G\left(y_{j}\right) \\
& =T A_{j-1} \lambda^{\prime}(T)=\widetilde{\mathcal{L}}_{\mathcal{A}, i}^{\prime}(T) .
\end{aligned}
$$

The inequality is due to $l<u \leq 0$ and $\mu_{y}<0$. Therefore, if the two policies adopt the same $N \geq 1$, then $\sum_{j=1}^{N} \mathcal{L}_{\mathcal{A}, i}(T)<$ $\sum_{j=1}^{N} \widetilde{\mathcal{L}}_{\mathcal{A}, i}(T)$ for all $T>-l$.

Moreover, as the feasible domain of PM interval $T$ in the unpunctual scenario is $T>-l$, there are two possible cases:

(a) if $\sum_{j=1}^{N} \widetilde{\mathcal{L}}_{\mathcal{A}, i}(-l) \geq \frac{(N-1) c_{p}+c_{r}}{c_{m}}$, then $\widetilde{T}^{*}<-l$, which means that $\widetilde{T}^{*}<-l<T^{*}$;

(b) if $\sum_{j=1}^{N} \widetilde{\mathcal{L}}_{\mathcal{A}, i}(-l)<\frac{(N-1) c_{p}+c_{r}}{c_{m}}$, then both $T^{*}$ and $\widetilde{T}^{*}$ are greater than $-l$. Because $\sum_{j=1}^{N} \mathcal{L}_{\mathcal{A}, i}(T)<$ $\sum_{j=1}^{N} \widetilde{\mathcal{L}}_{\mathcal{A}, i}(T)$ for all $T>-l$, it is clear that $-l<$ $\widetilde{T}^{*}<T^{*}$

Part (ii): In order to prove $N^{*} \geq \widetilde{N}^{*}$, what we need to show is that $\mathcal{J}_{\mathcal{A}}(T, N)<\widetilde{\mathcal{J}}_{\mathcal{A}}(T, N)$ for all $N \geq 1$. First of all, notice that

$$
\begin{aligned}
& \mathcal{J}_{\mathcal{A}}(T, N)-\mathcal{J}_{\mathcal{A}}(T, N-1) \\
& =N\left(A_{N}-A_{N-1}\right) \int_{l}^{u} \Lambda\left(T+y_{N}\right) \mathrm{d} G\left(y_{N}\right) \\
& <N\left(A_{N}-A_{N-1}\right) \Lambda(T) \\
& =\widetilde{\mathcal{J}}_{\mathcal{A}}(T, N)-\widetilde{\mathcal{J}}_{\mathcal{A}}(T, N-1) .
\end{aligned}
$$

The inequality is due to the negativity of $l$ and $u$. Hence, $\mathcal{J}_{\mathcal{A}}(T, N)$ increases slower than $\widetilde{\mathcal{J}}_{\mathcal{A}}(T, N)$. Coupled with the fact $\mathcal{J}_{\mathcal{A}}(T, 0)=\widetilde{\mathcal{J}}_{\mathcal{A}}(T, 0)=0$, we conclude that $\mathcal{J}_{\mathcal{A}}(T, N)<$ $\widetilde{\mathcal{J}}_{\mathcal{A}}(T, N)$ for all $N \geq 1$. Therefore, if the two policies specify the same $T>-l$, we have $N^{*} \geq \widetilde{N}^{*}$.

\section{REFERENCES}

[1] H. Wang, "A survey of maintenance policies of deteriorating systems," European Journal of Operational Research, vol. 139, no. 3, pp. 469-489, 2002 .

[2] S. Wu and M. J. Zuo, "Linear and nonlinear preventive maintenance models," IEEE Transactions on Reliability, vol. 59, no. 1, pp. 242-249, 2010.

[3] R. Barlow and L. Hunter, "Optimum preventive maintenance policies," Operations Research, vol. 8, no. 1, pp. 90-100, 1960.

[4] M. Finkelstein, M. Shafiee, and A. N. Kotchap, "Classical optimal replacement strategies revisited," IEEE Transactions on Reliability, vol. 65 , no. 2 , pp. 540-546, 2016.

[5] Z. Liang and A. K. Parlikad, "Predictive group maintenance for multisystem multi-component networks," Reliability Engineering \& System Safety, vol. 195, p. 106704, 2020.

[6] J. Shen, L. Cui, and Y. Ma, "Availability and optimal maintenance policy for systems degrading in dynamic environments," European Journal of Operational Research, vol. 276, no. 1, pp. 133-143, 2019.

[7] Q. Sun, Z.-S. Ye, and N. Chen, "Optimal inspection and replacement policies for multi-unit systems subject to degradation," IEEE Transactions on Reliability, vol. 67, no. 1, pp. 401-413, 2018.

[8] H. C. Vu, P. Do, M. Fouladirad, and A. Grall, "Dynamic opportunistic maintenance planning for multi-component redundant systems with various types of opportunities," Reliability Engineering \& System Safety, vol. 198, p. 106854, 2020.

[9] X. Zhu, X. Bei, N. Chatwattanasiri, and D. W. Coit, "Optimal system design and sequential preventive maintenance under uncertain aperiodicchanging stresses," IEEE Transactions on Reliability, vol. 67, no. 3, pp. 907-919, 2018. 
[10] T. Nakagawa, "Periodic and sequential preventive maintenance policies," Journal of Applied Probability, vol. 23, no. 2, pp. 536-542, 1986.

[11] H. Pham and H. Wang, "Imperfect maintenance," European Journal of Operational Research, vol. 94, no. 3, pp. 425-438, 1996.

[12] L. Doyen and O. Gaudoin, "Classes of imperfect repair models based on reduction of failure intensity or virtual age," Reliability Engineering \& System Safety, vol. 84, no. 1, pp. 45-56, 2004.

[13] G. J. Wang and R. C. Yam, "Generalized geometric process and its application in maintenance problems," Applied Mathematical Modelling, vol. 49, pp. 554-567, 2017.

[14] G.-J. Wang, Y.-L. Zhang, and R. C. Yam, "Preventive maintenance models based on the generalized geometric process," IEEE Transactions on Reliability, vol. 66, no. 4, pp. 1380-1388, 2017.

[15] T. Nakagawa, "Sequential imperfect preventive maintenance policies," IEEE Transactions on Reliability, vol. 37, no. 3, pp. 295-298, 1988.

[16] T. Nakagawa and S. Mizutani, "A summary of maintenance policies for a finite interval," Reliability Engineering \& System Safety, vol. 94, no. 1, pp. 89-96, 2009.

[17] M. Kijima, "Some results for repairable systems with general repair," Journal of Applied Probability, vol. 26, no. 1, pp. 89-102, 1989.

[18] D. Lin, M. J. Zuo, and R. C. M. Yam, "Sequential imperfect preventive maintenance models with two categories of failure modes," Naval Research Logistics (NRL), vol. 48, no. 2, pp. 172-183, 2001.

[19] S. Wu and D. Clements-Croome, "Preventive maintenance models with random maintenance quality," Reliability Engineering \& System Safety, vol. 90, no. 1, pp. 99-105, 2005.

[20] R. I. Zequeira and C. Bérenguer, "Periodic imperfect preventive maintenance with two categories of competing failure modes," Reliability Engineering \& System Safety, vol. 91, no. 4, pp. 460-468, 2006.

[21] S.-H. Sheu and C.-C. Chang, "An extended periodic imperfect preventive maintenance model with age-dependent failure type," IEEE Transactions on Reliability, vol. 58, no. 2, pp. 397-405, 2009.

[22] J. H. Cha and M. Finkelstein, "Optimal long-run imperfect maintenance with asymptotic virtual age," IEEE Transactions on Reliability, vol. 65 , no. 1, pp. 187-196, 2016

[23] Q. Sun, Z.-S. Ye, and W. Peng, "Scheduling preventive maintenance considering the saturation effect," IEEE Transactions on Reliability, vol. 68 , no. 2, pp. 741-752, 2019.

[24] X. Zhao, O. Gaudoin, L. Doyen, and M. Xie, "Optimal inspection and replacement policy based on experimental degradation data with covariates," IISE Transactions, vol. 51, no. 3, pp. 322-336, 2019.

[25] D. N. P. Murthy and N. Jack, Extended Warranties, Maintenance Service and Lease Contracts: Modeling and Analysis for Decision-Making. London, UK: Springer, 2014.

[26] Y.-H. Chien, "The optimal preventive-maintenance policy for a NHPBP repairable system under free-repair warranty," Reliability Engineering \& System Safety, vol. 188, pp. 444-453, 2019.

[27] L. Shang, S. Si, S. Sun, and T. Jin, "Optimal warranty design and postwarranty maintenance for products subject to stochastic degradation," IISE Transactions, vol. 50, no. 10, pp. 913-927, 2018.

[28] C. Su and X. Wang, "A two-stage preventive maintenance optimization model incorporating two-dimensional extended warranty," Reliability Engineering \& System Safety, vol. 155, pp. 169-178, 2016.

[29] X. Wang, L. Li, and M. Xie, "Optimal preventive maintenance strategy for leased equipment under successive usage-based contracts," International Journal of Production Research, vol. 57, no. 18, pp. 5705-5724, 2019.

[30] Q. Qiu, L. Cui, and L. Yang, "Maintenance policies for energy systems subject to complex failure processes and power purchasing agreement," Computers \& Industrial Engineering, vol. 119, pp. 193-203, 2018.

[31] S. Petchrompo, H. Li, A. K. Parlikad, A. Erguido, and C. Riches, "A value-based approach to optimising long-term maintenance plans for a multi-asset k-out-of-n system," Reliability Engineering \& System Safety, p. in press, 2020.

[32] B. de Jonge, A. S. Dijkstra, and W. Romeijnders, "Cost benefits of postponing time-based maintenance under lifetime distribution uncertainty," Reliability Engineering \& System Safety, vol. 140, pp. 15-21, 2015.

[33] L. Yang, Z.-S. Ye, C.-G. Lee, S.-F. Yang, and R. Peng, "A twophase preventive maintenance policy considering imperfect repair and postponed replacement," European Journal of Operational Research, vol. 274, no. 3, pp. 966-977, 2019.

[34] P. A. Scarf, C. A. V. Cavalcante, and R. S. Lopes, "Delay-time modelling of a critical system subject to random inspections," European Journal of Operational Research, vol. 278, no. 3, pp. 772-782, 2019.

[35] X. Zhao, T. Nakagawa, and M. J. Zuo, "Optimal replacement last with continuous and discrete policies," IEEE Transactions on Reliability, vol. 63 , no. 4 , pp. 868-880, 2014.
[36] X. Zhao, K. N. Al-Khalifa, A. M. Hamouda, and T. Nakagawa, "First and last triggering event approaches for replacement with minimal repairs," IEEE Transactions on Reliability, vol. 65, no. 1, pp. 197-207, 2016.

[37] Z. Zhu, Y. Xiang, M. Li, W. Zhu, and K. Schneider, "Preventive maintenance subject to equipment unavailability," IEEE Transactions on Reliability, vol. 68, no. 3, pp. 1009-1020, 2019.

[38] K. He, L. M. Maillart, and O. A. Prokopyev, "Optimal planning of unpunctual preventive maintenance," IISE Transactions, vol. 49, no. 2, pp. 127-143, 2017.

[39] X. Wang, L. Li, and M. Xie, "An unpunctual preventive maintenance policy under two-dimensional warranty," European Journal of Operational Research, vol. 282, no. 1, pp. 304-318, 2020.

[40] R. E. Barlow and F. Proschan, Mathematical Theory of Reliability. New York, USA: Wiley, 1965.

Xiaolin Wang (S'19) received the B.E. and M.S. degrees in industrial engineering from Southeast University, Nanjing, China, in 2013 and 2016, respectively. He is currently working towards the Ph.D. degree in industrial engineering with the Department of Systems Engineering and Engineering Management, City University of Hong Kong, Kowloon, Hong Kong.

His research interests include warranty analytics, maintenance planning, and degradation modeling, etc. His publications have appeared in journals such as IISE Transactions, European Journal of Operational Research, Reliability Engineering \& System Safety, International Journal of Production Research, Computers \& Industrial Engineering, etc.

He was a recipient of the prestigious Hong Kong $\mathrm{PhD}$ Fellowship from the Hong Kong Research Grants Council in 2017.

\section{Hang Zhou}

Ajith Kumar Parlikad ( $\mathrm{M}^{* * *}$ ) received the Ph.D. degree in manufacturing management from Cambridge University, Cambridge, U.K., in 2006.

$\mathrm{He}$ is currently Reader in Asset Management at the Department of Engineering, University of Cambridge. $\mathrm{He}$ is based at the Institute for Manufacturing, where he is the Head of the Asset Management Research Group. His research focuses on examining how data and digital technologies can be exploited to improve through-life asset performance.

Dr. Parlikad is a member of the steering committees of the IFAC Working Group on Advanced Maintenance Engineering, Services, and Technology and the UK. Digital Twin Hub.

Min Xie (M'91-SM'94-F'06) received the Ph.D. degree in quality technology from Linkoping University, Linkoping, Sweden, in 1987.

$\mathrm{He}$ is currently a Chair Professor with Department of Systems Engineering and Engineering Management, City University of Hong Kong, Kowloon, Hong Kong. He has authored or coauthored about 300 peer-reviewed journal papers and eight books on quality and reliability engineering, including Computing System Reliability (Kluwer), Stochastic Aging and Dependence for Reliability (Springer), and Weibull Models (Wiley). More than 50 Ph.D. students have graduated under his supervision.

Dr. Xie has chaired many international conferences and given keynote speeches. He also serves as an Editor and Associate Editor and on the Editorial Board of many established international journals. He was the recipient of the prestigious Lee Kuan Yew (LKY) Research Fellowship in 1991. 